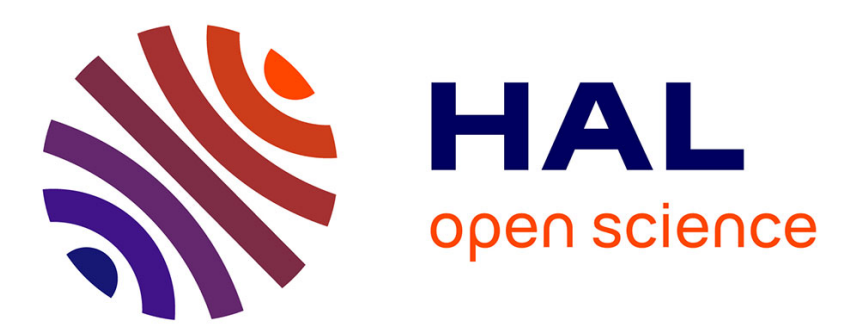

\title{
Weighted Hilbert spaces for the Stationary exterior Stokes problem with Navier slip boundary conditions
}

Anis Dhifaoui, Mohamed Meslameni, Ulrich Razafison

\section{To cite this version:}

Anis Dhifaoui, Mohamed Meslameni, Ulrich Razafison. Weighted Hilbert spaces for the Stationary exterior Stokes problem with Navier slip boundary conditions. Journal of Mathematical Analysis and Applications, 2019, 472 (2), pp.1846-1871. 10.1016/j.jmaa.2018.12.026 . hal-01788047v3

\section{HAL Id: hal-01788047 \\ https://hal.science/hal-01788047v3}

Submitted on 23 Mar 2019

HAL is a multi-disciplinary open access archive for the deposit and dissemination of scientific research documents, whether they are published or not. The documents may come from teaching and research institutions in France or abroad, or from public or private research centers.
L'archive ouverte pluridisciplinaire HAL, est destinée au dépôt et à la diffusion de documents scientifiques de niveau recherche, publiés ou non, émanant des établissements d'enseignement et de recherche français ou étrangers, des laboratoires publics ou privés. 


\title{
Weighted Hilbert spaces for the Stationary exterior Stokes problem with Navier slip boundary conditions
}

\author{
Anis Dhifaoui $^{1,2} \quad$ Mohamed Meslameni $^{3,4} \quad$ Ulrich Razafison $^{1}$ \\ 1 - Laboratoire de Mathématiques de Besançon CNRS UMR 6623, \\ Université Bourgogne Franche-Comté, \\ 16 route de Gray, 25030 Besançon Cedex, France \\ 2 - Laboratory Stability and Control of systems, \\ Faculty of Sciences of Sfax-Tunisia \\ Route de Soukra, km 3.5, BP 1171 \\ 3000 Sfax - Tunisia \\ 3 - Preparatory Institute for engineering Studies of Sfax \\ University of Sfax - Tunisia \\ 4 - College of Arts and Science in Al-Mandaq \\ AL Baha University - Kingdom of Saudi Arabia \\ Email adresses: anis.dhifaoui@univ-fcomte.fr, medmeslameni@yahoo.fr, \\ ulrich.razafison@univ-fcomte.fr
}

\begin{abstract}
In this paper, we analyse the three-dimensional exterior Stokes problem with the Navier slip boundary conditions, describing the flow of a viscous and incompressible fluid past an obstacle where it is assumed that the fluid may slip at the boundary. Because the flow domain is unbounded, we set the problem in weighted spaces in order to control the behavior at infinity of the solutions. This functional framework also allows to prescribe various behaviors at infinity of the solutions (growth or decay). Existence and uniqueness of solutions are shown in a Hilbert setting which gives the tools for a possible numerical analysis of the problem. Weighted Korn's inequalities are the key point in order to study the variational problem.
\end{abstract}

Keywords: Fluid Mechanics, Stokes equations, Navier slip boundary conditions, exterior domain, weighted spaces, Korn's inequalities.

AMS Subjets Classification: 76D07, 35J25, 76D03, 35J50.

\section{Introduction}

It is well-known that the motion of a viscous and incompressible fluid past an obstacle is governed by the Navier-Stokes equations. In the stationary case, the system can be written as follows:

$$
\begin{aligned}
& -v \Delta \boldsymbol{u}+\boldsymbol{u} \cdot \nabla \boldsymbol{u}+\nabla \pi=\boldsymbol{f} \text { in } \Omega \text {, } \\
& \operatorname{div} \boldsymbol{u}=0 \quad \text { in } \Omega \text {. }
\end{aligned}
$$

In (1.1), the flow domain $\Omega$ is assumed to be a three-dimensional simply connected exterior domain, namely the complement of a simply connected bounded domain which represents the obstacle, the unkowns are $\boldsymbol{u}$ the velocity of the fluid and its pressure $\pi$ and the data are $v$ the viscosity of the fluid and $\boldsymbol{f}$ the external forces acting on the fluid. 
Due to the fact that the flow domain $\Omega$ is unbounded, we need to add a condition at infinity. Here we assume that the fluid is at rest at infinity, therefore we append the following condition:

$$
\lim _{|\boldsymbol{x}| \rightarrow+\infty} \boldsymbol{u}(\boldsymbol{x})=\mathbf{0}
$$

In order to close the system, we also need a condition at the boundary $\Gamma$ of $\Omega$. Usually, the no-slip condition is prescribed for (1.1):

$$
\boldsymbol{u}=\mathbf{0} \text { on } \Gamma \text {. }
$$

One essential step in order to investigate the Navier-Stokes equations is to start by studying linearized problems. Here, due to the condition at infinity (1.2), the linearization leads to the Stokes equations:

$$
\begin{aligned}
-v \Delta \boldsymbol{u}+\nabla \pi=\boldsymbol{f} & \text { in } \quad \Omega, \\
\operatorname{div} \boldsymbol{u}=0 & \text { in } \Omega .
\end{aligned}
$$

Problem (1.4)-(1.3) in three-dimensional exterior domains has been studied by many authors. Among the works, we can mention $[5,24,25,26,29,30,32,38,39,49,50]$ and references therein. Note that the approach developed in $[5,29,30,32,49,50]$ is the same as the one we use in this paper.

However, in many applications, obstacles may have rough boundaries. This is the case, for instance, for the skin of sharks $([22,23])$ or golf balls. Another application of interest can also be found for instance in aerodynamics in drag control of aircraft wings. In order to reduce the drag, small injections jets are introduced over the wings of the plane ([3]).

In the case where the obstacles have a rough boundary, the no-slip condition (1.3) is no longer valid (see for instance [47]). Therefore, another approach was introduced where it is assumed that, due to the roughness of the boundary and to the viscosity of the fluid, there is a stagnant layer of fluid close to the boundary allowing the fluid to slip (see for instance [19]). To overcome the complicate description of the problem, the Navier slip boundary conditions are commonly used. These conditions, proposed by Navier [45], can be written as

$$
\boldsymbol{u} \cdot \boldsymbol{n}=0, \quad 2[\mathbf{D}(\boldsymbol{u}) \boldsymbol{n}]_{\tau}+\alpha \boldsymbol{u}_{\tau}=\mathbf{0} \quad \text { on } \quad \Gamma,
$$

where

$$
\mathbf{D}(\boldsymbol{u})=\frac{1}{2}\left(\nabla \boldsymbol{u}+\nabla \boldsymbol{u}^{T}\right)
$$

denotes the rate-of-strain tensor field, $\alpha$ is a scalar friction function, $\boldsymbol{n}$ is the unit normal vector to $\Gamma$ and the notation $[\cdot]_{\tau}$ denotes the tangential component of a vector on $\Gamma$. The first condition in (1.5) is the no-penetration condition and the second condition expresses the fact that the tangential velocity, instead of being zero as in the slip condition (1.3), is proportional to the tangential stress.

The Navier slip conditions have been extensively studied, see for instance [1, 2, 12, 19, 27, 35, 36, 48] and references therein.

The purpose of this paper is to study the exterior problem composed by the Stokes equations (1.4) and the Navier slip boundary conditions (1.5), with a positive friction function $\alpha$ and where we also include the non homogeneous case. Although the Stokes problem set in bounded domains with conditions (1.5) has been well studied by various authors (see for instance $[10,13]$ or $[11,48]$ for the case $\alpha=0$ and references therein), to the best of our knowledge, it is not the case when the domain is unbounded and $\alpha>0$. We can just mention [46] where (1.5) was used for the stationary Navier-Stokes equations in exterior domains with also the assumption that the velocity tends to a non zero constant vector at infinity instead of (1.2). For the case of Navier boundary conditions without friction ( $\alpha=0$ ), let us mention [9], where the following boundary conditions (also known as Hodge boundary conditions) were used:

$$
\boldsymbol{u} \cdot \boldsymbol{n}=\mathbf{0}, \quad \operatorname{curl} \boldsymbol{u} \times \boldsymbol{n}=\mathbf{0} \quad \text { on } \quad \Gamma,
$$


where curl $\boldsymbol{u}$ is the vorticity field. These conditions coincide with (1.5) on flat boundaries when $\alpha=0$. They were also used in [14] for the study of the non stationary Navier-Stokes equations in half-spaces of $\mathbb{R}^{3}$. We finally refer to $[43,44]$ for the study of the non stationary problem of Navier-Stokes with mixed boundary conditions that include (1.5) without friction.

This paper is the starting point of our investigation of problem (1.4)-(1.5). The study is based on a $L^{2}$ theory and, because the domain $\Omega$ is unbounded, we choose to set the problem in weighted spaces. The weight functions are polynomials and enable to describe the growth or the decay of functions at infinity which allows to look for solutions of (1.4)-(1.5) with various behavior at infinity and this is one of the main advantages of the weighted spaces. Moreover, a numerical method, the so-called inverted finite elements method, that uses weighted spaces as functional framework has been developed in the pioneer work [16]. Hence, this method allows to approximate the various solutions found in the weighted spaces framework. Note that the finite elements method has been efficiently applied to many elliptic problems set in unbounded domains (see for instance [15, 17, 18]).

Our analysis of problem (1.4)-(1.5) follows the same lines as in [33] for the study of the exterior Laplace problems or [32] and [29] for the exterior Stokes problem with no-slip boundary conditions: we first look for the variational solution. The key point here is the proof of variant weighted Korn's inequalities in order to get the coercivity of the bilinear form associated to the variational formulation. To establish these inequalities, we follow the approach introduced in [21], where the authors proposed another proof of Korn's inequalities in bounded domains based on the fact that symmetric tensor fields that satisfy the so-called Saint-Venant compatibility condition (see (2.22)), can be written as the rate-of-strain tensor of a vector field. Let us mention that other variant weighted Korn's inequalities can be found for instance in [37]. Next, we look for solutions that have a different behavior at infinity from the variational solution. To that end, we combine results on the Stokes problem set in the whole space to catch the behavior at infinity and results in bounded domains to take into account the boundary conditions. We start by looking for solutions that grow at infinity or decay weaker than the variational solution. But afterwards, unlike the exterior problem of Laplace with Dirichlet boundary or problem (1.4)-(1.3), duality arguments can not be applied in our context to find solutions that decay faster than the variational solution. Instead we use arguments that are similar as proving regularity results. Here, the arguments are based on adequate extensions of solutions to (1.4)-(1.5) that we already have at hand. These techniques were previoulsy applied in [33] (see also [42]) for the exterior Laplace equation with Neumann boundary condition.

The paper is organized as follows. In Section 2, we introduce the Notation, the functional framework based on weighted Hilbert spaces. We shall precise in which sense, the Navier slip boundary conditions are taken. We recall the main results on Stokes problem that we shall use and we establish the Korn's inequalities. In Section 3, we solve problem (1.4)-(1.5) by following the same lines described above.

\section{Notation and preliminaries}

\subsection{Notation}

Throughout this paper we assume that $\Omega^{\prime} \subset \mathbb{R}^{3}$ is a simply connected bounded domain that has at least a Lipschitz-continuous boundary $\Gamma$. Let $\Omega$ be the complement of $\overline{\Omega^{\prime}}$ in $\mathbb{R}^{3}$, in other words an exterior domain. We will use bold characters for vector and matrix fields. A point in $\mathbb{R}^{3}$ is denoted by $\boldsymbol{x}=\left(x_{1}, x_{2}, x_{3}\right)$ and its distance to the origin by

$$
r=|\boldsymbol{x}|=\left(x_{1}^{2}+x_{2}^{2}+x_{3}^{2}\right)^{1 / 2} .
$$


Let $\mathbb{N}$ denote the set of non-negative integers and $\mathbb{Z}$ the set of all integers . For any multi-index $\boldsymbol{\lambda} \in \mathbb{N}^{3}$, we denote by $\partial^{\lambda}$ the differential operator of order $\lambda$,

$$
\partial^{\lambda}=\frac{\partial^{|\lambda|}}{\partial_{1}^{\lambda_{1}} \partial_{2}^{\lambda_{2}} \partial_{3}^{\lambda_{3}}}, \quad|\boldsymbol{\lambda}|=\lambda_{1}+\lambda_{2}+\lambda_{3} .
$$

For any $k \in \mathbb{Z}, \mathscr{P}_{k}$ stands for the space of polynomials of degree less than or equal to $k$ and $\mathscr{P}_{k}^{\Delta}$ the harmonic polynomials of $\mathscr{P}_{k}$. If $k$ is a negative integer, we set by convention $\mathscr{P}_{k}=\{0\}$. We denote by $\mathscr{D}(\Omega)$ the space of $\mathscr{C}^{\infty}$ functions with compact support in $\Omega, \mathscr{D}(\bar{\Omega})$ the restriction to $\Omega$ of functions belonging to $\mathscr{D}\left(\mathbb{R}^{3}\right)$. We recall that $\mathscr{D}^{\prime}(\Omega)$ is the well-known space of distributions defined on $\Omega$. We recall that $L^{p}(\Omega)$ is the well-known Lebesgue real space and for $m \geq 1$, we recall that $H^{m}(\Omega)$ is the wellknown Hilbert space $W^{m, 2}(\Omega)$. We shall write $u \in H_{l o c}^{m}(\Omega)$ to mean that $u \in H^{m}(\mathscr{O})$, for any bounded domain $\mathscr{O}$, with $\bar{O} \subset \Omega$. For $R>0$, we denote by $B_{R}$ the open ball of radius $R$ centered at $\mathbf{0}$. We assume that the origin of the coordinates is placed in the obstacle $\Omega^{\prime}$. We set $\Omega_{R}=\Omega \cap B_{R}$. The notation $\langle\cdot, \cdot\rangle$ will denote adequate duality pairing and will be specified when needed. If not specified, $\langle\cdot, \cdot\rangle_{\Gamma}$ will denote the duality pairing between the space $H^{-1 / 2}(\Gamma)$ and its dual space $H^{1 / 2}(\Gamma)$. Given $\boldsymbol{A}$ and $\boldsymbol{B}$ two matrix fields, such that $\boldsymbol{A}=\left(a_{i j}\right)_{1 \leqslant i, j \leqslant 3}$ and $\boldsymbol{B}=\left(b_{i j}\right)_{1 \leqslant i, j \leqslant 3}$, then we define $\boldsymbol{A}: \boldsymbol{B}=\left(a_{i j} b_{i j}\right)_{1 \leqslant i, j \leqslant 3}$. Finally, as usual, $C>0$ denotes a generic constant the value of which may change from line to line and even at the same line.

\subsection{Weighted Hilbert spaces}

We introduce the weight function

$$
\rho(\boldsymbol{x})=\left(1+r^{2}\right)^{1 / 2}
$$

For $k \in \mathbb{Z}$, we introduce

$$
W_{k}^{0}(\Omega)=\left\{u \in \mathscr{D}^{\prime}(\Omega), \rho^{k} u \in L^{2}(\Omega)\right\},
$$

which is a Hilbert space equipped with the norm:

$$
\|u\|_{W_{k}^{0}(\Omega)}=\left\|\rho^{k} u\right\|_{L^{2}(\Omega)} .
$$

Let $m \geqslant 1$ be an integer. We define the weighted Hilbert space:

$$
W_{k}^{m}(\Omega)=\left\{u \in \mathscr{D}^{\prime}(\Omega) ; \forall \boldsymbol{\lambda} \in \mathbb{N}^{3}: 0 \leq|\boldsymbol{\lambda}| \leq m, \rho^{k-m+|\boldsymbol{\lambda}|} \partial^{\lambda} u \in L^{2}(\Omega)\right\},
$$

equipped with the norm

$$
\|u\|_{W_{k}^{m}(\Omega)}=\left(\sum_{0 \leqslant|\lambda| \leqslant m}\left\|\rho^{k-m+|\lambda|} \partial^{\lambda} u\right\|_{L^{2}(\Omega)}^{2}\right)^{1 / 2} .
$$

We define the semi-norm

$$
|u|_{W_{k}^{m}(\Omega)}=\left(\sum_{|\lambda|=m}\left\|\rho^{k} \partial^{\lambda} u\right\|_{L^{2}(\Omega)}\right)^{1 / 2} .
$$

Let us give an example of such space that will be often used in the remaining of the paper. For $m=1$, we have

$$
W_{k}^{1}(\Omega)=\left\{u \in \mathscr{D}^{\prime}(\Omega), \rho^{k-1} u \in L^{2}(\Omega), \rho^{k} \nabla u \in L^{2}(\Omega)\right\} .
$$

For the sake of simplicity, we have defined these spaces with integer exponents on the weight function. But naturally, these definitions can be extended to real number exponents with eventually some slight modifications (see[7] for more details).

We shall now give some basic properties of those spaces:

\section{Properties 2.1.}


1. The space $\mathscr{D}(\bar{\Omega})$ is dense in $W_{k}^{m}(\Omega)$.

2. For any $\boldsymbol{\lambda} \in \mathbb{N}^{3}$, the mapping

$$
u \in W_{k}^{m}(\Omega) \rightarrow \partial^{\lambda} u \in W_{k}^{m-|\lambda|}(\Omega)
$$

is continuous.

3. For $m \in \mathbb{N} \backslash\{0\}$ and $k \in \mathbb{Z}$, we have the following continuous imbedding:

$$
W_{k}^{m}(\Omega) \hookrightarrow W_{k-1}^{m-1}(\Omega) .
$$

4. The space $\mathscr{P}_{m-2-k}$ is the space of all polynomials included in $W_{k}^{m}(\Omega)$ and the following Poincarétype inequality holds:

$$
\forall u \in W_{k}^{m}(\Omega), \quad \inf _{\mu \in \mathscr{P}_{j^{\prime}}}\|u+\mu\|_{W_{k}^{m}(\Omega)} \leqslant C|u|_{W_{k}^{m}(\Omega)},
$$

where $j^{\prime}=\min (m-2-k, m-1)$. In other words the semi-norm $|\cdot|_{W_{k}^{m}(\Omega)}$ is a norm on $W_{k}^{m}(\Omega) / \mathscr{P}_{j^{\prime}}$. In particular, $|\cdot|_{W_{0}^{1}(\Omega)}$ is a norm on $W_{0}^{1}(\Omega)$.

For more details on the above properties and the ones that we shall present in the following, the reader can refer to $[7,8,34,40]$ and references therein.

Note that all the local properties of the space $W_{k}^{m}(\Omega)$ coincide with those of the standard Hilbert space $H^{m}(\Omega)$. Hence, it also satisfies the usual trace theorems on the boundary $\Gamma$. Therefore, we can define the space

$$
\stackrel{\circ}{W}_{k}^{m}(\Omega)=\left\{u \in W_{k}^{m}(\Omega), \gamma_{0} u=0, \gamma_{1} u=0, \cdots, \gamma_{m-1} u=0 \text { on } \Gamma\right\} .
$$

If $\Omega$ is the whole space $\mathbb{R}^{3}$, then the spaces $\stackrel{\circ}{W}_{k}^{m}\left(\mathbb{R}^{3}\right)$ and $W_{k}^{m}\left(\mathbb{R}^{3}\right)$ coincide. The space $\mathscr{D}(\Omega)$ is dense in $\stackrel{\circ}{W}_{k}^{m}(\Omega)$. Therefore, the dual space of $\stackrel{\circ}{W}_{k}^{m}(\Omega)$, denoted by $W_{-k}^{-m}(\Omega)$ is a space of distributions. For $m \in \mathbb{N}$ and $k \in \mathbb{Z}$, we have the continuous imbedding

$$
W_{k}^{-m}(\Omega) \subset W_{k-1}^{-m-1}(\Omega) .
$$

Moreover, we have the following Poincaré-type inequality:

$$
\forall u \in \stackrel{\circ}{W}_{k}^{m}(\Omega), \quad\|u\|_{W_{k}^{m}(\Omega)} \leq C|u|_{W_{k}^{m}(\Omega)} .
$$

We now introduce some weighted Hilbert spaces that are specific for the study of the Stokes problem (1.4) with the Navier boundary conditions (1.5). We start by introducing the following space for $k \in \mathbb{Z}:$

$$
H_{k}(\operatorname{div} ; \Omega)=\left\{\boldsymbol{v} \in W_{k}^{0}(\Omega), \operatorname{div} \boldsymbol{v} \in W_{k+1}^{0}(\Omega)\right\} .
$$

This space is endowed with the norm

$$
\|\boldsymbol{v}\|_{H_{k}(\operatorname{div} ; \Omega)}=\left(\|\boldsymbol{v}\|_{W_{k}^{0}(\Omega)}^{2}+\|\operatorname{div} \boldsymbol{v}\|_{W_{k+1}^{0}(\Omega)}^{2}\right)^{1 / 2} .
$$

Observe that $\mathscr{D}(\bar{\Omega})$ is dense in $H_{k}(\operatorname{div} ; \Omega)$. For the proof, one can use the same arguments as for the proof of the density of $\mathscr{D}(\bar{\Omega})$ in $W_{k}^{m}(\Omega)$ (see [34]). Therefore, denoting by $\boldsymbol{n}$ the unit normal vector to the boundary $\Gamma$ pointing outside $\Omega$, if $\boldsymbol{v}$ belongs to $H_{k}(\operatorname{div} ; \Omega)$, then $\boldsymbol{v}$ has a normal trace $\boldsymbol{v} \cdot \boldsymbol{n}$ in $H^{-1 / 2}(\Gamma)$ and there exists $C>0$ such that

$$
\forall \boldsymbol{v} \in H_{k}(\operatorname{div} ; \Omega), \quad\|\boldsymbol{v} \cdot \boldsymbol{n}\|_{H^{-1 / 2}(\Gamma)} \leq C\|\boldsymbol{v}\|_{H_{k}(\operatorname{div} ; \Omega)} .
$$


Moreover the below Green's formula holds. For any $\boldsymbol{v} \in H_{k}(\operatorname{div} ; \Omega)$ and $\varphi \in W_{-k}^{1}(\Omega)$, we have

$$
\langle\boldsymbol{v} \cdot \boldsymbol{n}, \varphi\rangle_{\Gamma}=\int_{\Omega} \boldsymbol{v} \cdot \nabla \varphi d \boldsymbol{x}+\int_{\Omega} \varphi \operatorname{div} \boldsymbol{v} d \boldsymbol{x} .
$$

The closure of $\mathscr{D}(\Omega)$ in $H_{k}(\operatorname{div} ; \Omega)$ is denoted by $\stackrel{\circ}{H}_{k}(\operatorname{div} ; \Omega)$ and can be characterized by

$$
\stackrel{\circ}{H}_{k}(\operatorname{div} ; \Omega)=\left\{\boldsymbol{v} \in H_{k}(\operatorname{div} ; \Omega), \boldsymbol{v} \cdot \boldsymbol{n}=0 \text { on } \Gamma\right\} .
$$

Its dual space is denoted by $H_{-k}^{-1}(\operatorname{div} ; \Omega)$ and is characterized by the below proposition.

Proposition 2.2. Assume that $\Omega$ is of class $\mathscr{C}^{1,1}$ and let $k \in \mathbb{Z}$. A distribution fbelongs to $H_{k}^{-1}(\operatorname{div} ; \Omega)$ if and only if there exist $\boldsymbol{\psi} \in W_{k}^{0}(\Omega)$ and $\chi \in W_{k-1}^{0}(\Omega)$, such that $\boldsymbol{f}=\boldsymbol{\psi}+\nabla \chi$. Moreover

$$
\|\boldsymbol{\psi}\|_{W_{k}^{0}(\Omega)}+\|\chi\|_{W_{k-1}^{0}(\Omega)} \leq C\|\boldsymbol{f}\|_{H_{k}^{-1}(\mathrm{div} ; \Omega)} .
$$

The proof of Proposition 2.2 can be found in [9, Proposition 1.3]. A consequence of this proposition and the imbedding (2.4) is that, for any $k \in \mathbb{Z}$, we have the imbedding

$$
H_{k}^{-1}(\operatorname{div} ; \Omega) \subset W_{k-1}^{-1}(\Omega) .
$$

\subsection{The Stokes equations in the whole space $\mathbb{R}^{3}$}

We recall here some basic results concerning the Stokes problem in $\mathbb{R}^{3}$ :

$$
-\Delta \boldsymbol{u}+\nabla \pi=\boldsymbol{f} \text { and } \operatorname{div} \boldsymbol{u}=\chi \text { in } \mathbb{R}^{3} .
$$

These results can be found in [28] (see also [4] for an extension to weighted $L^{p}$ spaces). Let us first introduce the kernel of the Stokes operator

$$
\mathscr{N}_{k}\left(\mathbb{R}^{3}\right)=\left\{(\boldsymbol{u}, \pi) \in W_{k}^{1}\left(\mathbb{R}^{3}\right) \times W_{k}^{0}\left(\mathbb{R}^{3}\right),-\Delta \boldsymbol{u}+\nabla \pi=\mathbf{0} \text { and } \operatorname{div} \boldsymbol{u}=0 \text { in } \mathbb{R}^{3}\right\}
$$

and the space of polynomials

$$
N_{k}=\left\{(\boldsymbol{\lambda}, \mu) \in \mathscr{P}_{k} \times \mathscr{P}_{k-1}^{\Delta},-\Delta \boldsymbol{\lambda}+\nabla \mu=\mathbf{0} \text { and } \operatorname{div} \boldsymbol{\lambda}=0\right\} .
$$

Recall that by agreement on the notation $\mathscr{P}_{k}$, the space $N_{k}=\{(\mathbf{0}, 0)\}$ when $k<0$ and $N_{0}=\mathscr{P}_{0} \times\{0\}$. The next proposition characterizes the kernel of (2.9).

\section{Proposition 2.3.}

- If $k \geqslant 0$, then $\mathscr{N}_{k}\left(\mathbb{R}^{3}\right)=\{(\mathbf{0}, 0)\}$.

- If $k<0$, then $\mathscr{N}_{k}\left(\mathbb{R}^{3}\right)=N_{-k-1}$.

The next theorem states an existence, uniqueness and regularity result for problem (2.9).

Theorem 2.4. Assume that $k \in \mathbb{Z}$. If $(f, \chi) \in W_{k}^{-1}\left(\mathbb{R}^{3}\right) \times W_{k}^{0}\left(\mathbb{R}^{3}\right)$ satisfies the compatibility condition:

$$
\forall(\boldsymbol{\lambda}, \mu) \in N_{k-1}, \quad\langle\boldsymbol{f}, \boldsymbol{\lambda}\rangle_{W_{k}^{-1}\left(\mathbb{R}^{3}\right) \times W_{-k}^{1}\left(\mathbb{R}^{3}\right)}-\langle\chi, \mu\rangle_{W_{k}^{0}\left(\mathbb{R}^{3}\right) \times W_{-k}^{0}\left(\mathbb{R}^{3}\right)}=0,
$$

then problem (2.9) has a solution $(\boldsymbol{u}, \pi) \in W_{k}^{1}\left(\mathbb{R}^{3}\right) \times W_{k}^{0}\left(\mathbb{R}^{3}\right)$ unique up to an element of $N_{-k-1}$ and we have the estimate

$$
\inf _{(\boldsymbol{\lambda}, \mu) \in N_{-k-1}}\left(\|\boldsymbol{u}+\boldsymbol{\lambda}\|_{W_{k}^{1}\left(\mathbb{R}^{3}\right)}+\|\pi+\mu\|_{W_{k}^{0}\left(\mathbb{R}^{3}\right)}\right) \leqslant C\left(\|\boldsymbol{f}\|_{W_{k}^{-1}\left(\mathbb{R}^{3}\right)}+\|\chi\|_{W_{k}^{0}\left(\mathbb{R}^{3}\right)}\right) .
$$

Furthermore, if $(\boldsymbol{f}, \chi) \in W_{k+1}^{0}\left(\mathbb{R}^{3}\right) \times W_{k+1}^{1}\left(\mathbb{R}^{3}\right)$, then $(\boldsymbol{u}, \pi) \in W_{k+1}^{2}\left(\mathbb{R}^{3}\right) \times W_{k+1}^{1}\left(\mathbb{R}^{3}\right)$. 


\subsection{The Navier boundary conditions and related properties}

Let us introduce some notations related to the boundary. First, for any vector field $\boldsymbol{v}$ on $\Gamma$, we can write

$$
\boldsymbol{v}=\boldsymbol{v}_{\tau}+(\boldsymbol{v} \cdot \boldsymbol{n}) \boldsymbol{n},
$$

where $\boldsymbol{v}_{\tau}$ is the projection of $\boldsymbol{v}$ on the tangent hyper-plan to $\Gamma$.

We need now to give a sense to the second condition in (1.5). To that end, we first introduce the spaces:

$$
V_{\sigma, T}(\Omega)=\left\{\boldsymbol{v} \in W_{0}^{1}(\Omega), \operatorname{div} \boldsymbol{v}=0 \text { in } \Omega \text { and } \boldsymbol{v} \cdot \boldsymbol{n}=0 \text { on } \Gamma\right\}
$$

and

$$
E(\Omega)=\left\{\boldsymbol{v} \in W_{0}^{1}(\Omega), \Delta \boldsymbol{v} \in H_{1}^{-1}(\operatorname{div} ; \Omega)\right\},
$$

equipped with the norm of $W_{0}^{1}(\Omega)$ and

$$
\|\boldsymbol{v}\|_{E(\Omega)}=\|\boldsymbol{v}\|_{W_{0}^{1}(\Omega)}+\|\Delta \boldsymbol{v}\|_{H_{1}^{-1}(\operatorname{div} ; \Omega)}
$$

respectively. We recall that the space $\mathscr{D}(\bar{\Omega})$ is dense in $E(\Omega)$ (see [9, Lemma 5.1]). As a consequence, we obtain the following trace result.

Lemma 2.5. The linear mapping $\Theta: \boldsymbol{u} \rightarrow[\boldsymbol{D}(\boldsymbol{u}) \boldsymbol{n}]_{\tau}$ defined on $\mathscr{D}(\bar{\Omega})$ can be extended to a linear and continuous mapping

$$
\Theta: E(\Omega) \rightarrow H^{-1 / 2}(\Gamma)
$$

Moreover, we have the Green formula: For any $\boldsymbol{u} \in E(\Omega)$ and $\boldsymbol{\varphi} \in V_{\sigma, T}(\Omega)$,

$$
-\langle\Delta \boldsymbol{u}, \boldsymbol{\varphi}\rangle_{\Omega}=2 \int_{\Omega} \boldsymbol{D}(\boldsymbol{u}): \boldsymbol{D}(\boldsymbol{\varphi}) d \boldsymbol{x}-2\left\langle[\boldsymbol{D}(\boldsymbol{u}) \boldsymbol{n}]_{\tau}, \boldsymbol{\varphi}\right\rangle_{\Gamma} .
$$

Where $\langle\cdot, \cdot\rangle_{\Omega}$ denotes the dualities between $H_{1}^{-1}(\operatorname{div} ; \Omega)$ and $\stackrel{\circ}{H}_{-1}(\operatorname{div} ; \Omega)$.

Proof. It is a standard proof that follows the ideas of [41]. The goal is to prove that the mapping $\Theta$ defined on $\mathscr{D}(\bar{\Omega})$ is continuous for the norm of $E(\Omega)$. Let us first observe that if $\boldsymbol{u} \in \mathscr{D}(\bar{\Omega})$ and $\boldsymbol{\varphi} \in$ $W_{0}^{1}(\Omega)$ with $\boldsymbol{\varphi} \cdot \boldsymbol{n}=0$ on $\Gamma$, then thanks to the following identity

$$
\Delta \boldsymbol{u}=2 \operatorname{div} \mathbf{D}(\boldsymbol{u})-\nabla \operatorname{div} \boldsymbol{u}
$$

and the Green formula (2.7), we have

$$
-\langle\Delta \boldsymbol{u}, \boldsymbol{\varphi}\rangle_{\Omega}=2 \int_{\Omega} \mathbf{D}(\boldsymbol{u}): \nabla \boldsymbol{\varphi} d \boldsymbol{x}-2\langle\mathbf{D}(\boldsymbol{u}) \boldsymbol{n}, \boldsymbol{\varphi}\rangle_{\Gamma}-\int_{\Omega} \operatorname{div} \boldsymbol{u} \operatorname{div} \boldsymbol{\varphi} d \boldsymbol{x} .
$$

Next, the fact that $\boldsymbol{\varphi} \cdot \boldsymbol{n}=0$ on $\Gamma$ also implies that

$$
\langle\mathbf{D}(\boldsymbol{u}) \boldsymbol{n}, \boldsymbol{\varphi}\rangle_{\Gamma}=\left\langle([\mathbf{D}(\boldsymbol{u}) \boldsymbol{n}] \cdot \boldsymbol{n}) \boldsymbol{n}+[\mathbf{D}(\boldsymbol{u}) \boldsymbol{n}]_{\tau}, \boldsymbol{\varphi}\right\rangle_{\Gamma}=\left\langle[\mathbf{D}(\boldsymbol{u}) \boldsymbol{n}]_{\tau}, \boldsymbol{\varphi}\right\rangle_{\Gamma} .
$$

It follows that, for any $\boldsymbol{u} \in \mathscr{D}(\bar{\Omega})$ and $\boldsymbol{\varphi} \in V_{\sigma, T}(\Omega)$, we have

$$
-\langle\Delta \boldsymbol{u}, \boldsymbol{\varphi}\rangle_{\Omega}=2 \int_{\Omega} \mathbf{D}(\boldsymbol{u}): \nabla \boldsymbol{\varphi} d \boldsymbol{x}-2\left\langle[\mathbf{D}(\boldsymbol{u}) \boldsymbol{n}]_{\tau}, \boldsymbol{\varphi}\right\rangle_{\Gamma} .
$$

Finally, since we have

$$
\int_{\Omega} \mathbf{D}(\boldsymbol{u}): \nabla \boldsymbol{\varphi} d \boldsymbol{x}=\int_{\Omega} \mathbf{D}(\boldsymbol{u}): \mathbf{D}(\boldsymbol{\varphi}) d \boldsymbol{x},
$$

we arrive at

$$
-\langle\Delta \boldsymbol{u}, \boldsymbol{\varphi}\rangle_{\Omega}=2 \int_{\Omega} \mathbf{D}(\boldsymbol{u}): \mathbf{D}(\boldsymbol{\varphi}) d \boldsymbol{x}-2\left\langle[\mathbf{D}(\boldsymbol{u}) \boldsymbol{n}]_{\tau}, \boldsymbol{\varphi}\right\rangle_{\Gamma}
$$


Let now $\boldsymbol{\mu}$ be any element of $H^{1 / 2}(\Gamma)$. Then there exists an element $\boldsymbol{\varphi}$ in $W_{0}^{1}(\Omega)$ such that $\operatorname{div} \boldsymbol{\varphi}=0$ in $\Omega$ and $\boldsymbol{\varphi}=\boldsymbol{\mu}_{\tau}$ on $\Gamma$ with the estimate

$$
\|\boldsymbol{\varphi}\|_{W_{0}^{1}(\Omega)} \leqslant C\left\|\boldsymbol{\mu}_{\tau}\right\|_{H^{1 / 2}(\Gamma)} \leqslant C\|\boldsymbol{\mu}\|_{H^{1 / 2}(\Gamma)} .
$$

Consequently, using (2.17), we can write

$$
\begin{aligned}
\left|2\left\langle[\mathbf{D}(\boldsymbol{u}) \boldsymbol{n}]_{\tau}, \boldsymbol{\mu}\right\rangle_{\Gamma}\right| & =\left|2\left\langle[\mathbf{D}(\boldsymbol{u}) \boldsymbol{n}]_{\tau}, \boldsymbol{\mu}_{\tau}\right\rangle_{\Gamma}\right|=\left|2\left\langle[\mathbf{D}(\boldsymbol{u}) \boldsymbol{n}]_{\tau}, \boldsymbol{\varphi}\right\rangle_{\Gamma}\right| \\
& \leqslant\|\Delta \boldsymbol{u}\|_{H_{1}^{-1}(\operatorname{div}, \Omega)}\|\boldsymbol{\varphi}\|_{H_{-1}(\operatorname{div}, \Omega)}+2\|\mathbf{D}(\boldsymbol{u})\|_{L^{2}(\Omega)}\|\mathbf{D}(\boldsymbol{\varphi})\|_{L^{2}(\Omega)} \\
& \leqslant\left(\|\Delta \boldsymbol{u}\|_{H_{1}^{-1}(\operatorname{div}, \Omega)}^{2}+2\|\mathbf{D}(\boldsymbol{u})\|_{L^{2}(\Omega)}^{2}\right)^{1 / 2} \times\left(\|\boldsymbol{\varphi}\|_{W_{0}^{1}(\Omega)}^{2}+2\|\mathbf{D}(\boldsymbol{\varphi})\|_{L^{2}(\Omega)}^{2}\right)^{1 / 2} \\
& \leqslant C\|\boldsymbol{u}\|_{E(\Omega)}\|\boldsymbol{\varphi}\|_{W_{0}^{1}(\Omega)} .
\end{aligned}
$$

Using inequalities (2.18) we deduce that

$$
\left|2\left\langle[\mathbf{D}(\boldsymbol{u}) \boldsymbol{n}]_{\tau}, \boldsymbol{\mu}\right\rangle_{\Gamma}\right| \leqslant C\|\boldsymbol{u}\|_{E(\Omega)}\|\boldsymbol{\mu}\|_{H^{1 / 2}(\Gamma)}
$$

which implies that

$$
\left\|[\mathbf{D}(\boldsymbol{u}) \boldsymbol{n}]_{\tau}\right\|_{H^{-1 / 2}(\Gamma)} \leqslant C\|\boldsymbol{u}\|_{E(\Omega)} .
$$

Therefore, the linear mapping $\Theta: \boldsymbol{u} \rightarrow[\mathbf{D}(\boldsymbol{u}) \boldsymbol{n}]_{\tau}$ defined in $\mathscr{D}(\bar{\Omega})$ is continuous for the norm of $E(\Omega)$. Since $\mathscr{D}(\bar{\Omega})$ is dense in $E(\Omega), \Theta$ can be extended by continuity to a mapping still called $\Theta$ defined on $E(\Omega)$ to $H^{-1 / 2}(\Gamma)$ and formula (2.14) holds for all $\boldsymbol{u} \in E(\Omega)$ and $\varphi \in V_{\sigma, T}(\Omega)$.

\subsection{Weighted Korn's inequalities}

This part is devoted to the proof of weighted Korn's inequalities by following the approach proposed by Ciarlet and Ciarlet in [21]. Let us first introduce some notations. For any vector fields $\boldsymbol{u}$ and $\boldsymbol{v}$ of $\mathbb{R}^{3}$, we define

$$
\boldsymbol{u} \times \boldsymbol{v}=\left(u_{2} v_{3}-v_{3} u_{2}, u_{3} v_{1}-u_{1} v_{3}, u_{1} v_{2}-u_{2} v_{1}\right)^{T}
$$

and

$$
\operatorname{curl} \boldsymbol{u}=\nabla \times \boldsymbol{u} .
$$

We start with a first lemma on a specific existence of vector potential.

Lemma 2.6. Suppose that $\Omega$ is of class $\mathscr{C}^{1,1}$ and let $k \in \mathbb{Z}$. Assume that $\boldsymbol{u}$ belongs to $W_{k}^{1}(\Omega)$ and satisfies $\operatorname{div} \boldsymbol{u}=0$ in $\Omega$. Then, there exists $\boldsymbol{\psi} \in \stackrel{\circ}{W}_{k}^{2}(\Omega)$ satisfying

$$
\boldsymbol{u}=\operatorname{curl} \boldsymbol{\psi} \text { in } \Omega \text {. }
$$

Proof. Denote by $\widetilde{\boldsymbol{u}}$ the extension of $\boldsymbol{u}$ by zero in $\Omega^{\prime}$. Then $\widetilde{\boldsymbol{u}}$ belongs to $W_{k}^{1}\left(\mathbb{R}^{3}\right)$ and satisfies $\operatorname{div} \widetilde{\boldsymbol{u}}=0$ in $\mathbb{R}^{3}$. Thanks to [28, Theorems 3.2 and 3.5] there exists $\widetilde{\boldsymbol{w}}$ that belongs to $W_{k}^{2}\left(\mathbb{R}^{3}\right)$ such that

$$
\widetilde{\boldsymbol{u}}=\operatorname{curl} \widetilde{\boldsymbol{w}} \text { in } \mathbb{R}^{3} .
$$

Denote now by $\boldsymbol{w}^{\prime}$ the restriction of $\widetilde{\boldsymbol{w}}$ on $\Omega^{\prime}$. Then curl $\boldsymbol{w}^{\prime}=\mathbf{0}$ in $\Omega^{\prime}$ and as a consequence $\boldsymbol{w}^{\prime}=\nabla \Phi^{\prime}$, where $\Phi^{\prime} \in H^{3}\left(\Omega^{\prime}\right)$ (see for instance [31]). Let $\widetilde{\Phi} \in W_{k}^{3}\left(\mathbb{R}^{3}\right)$ be an extension of $\Phi^{\prime}$ in the whole space $\mathbb{R}^{3}$ and let $\widetilde{\boldsymbol{\psi}} \in W_{k}^{2}\left(\mathbb{R}^{3}\right)$ be defined by $\widetilde{\boldsymbol{\psi}}=\widetilde{\boldsymbol{w}}-\nabla \widetilde{\Phi}$. We have

$$
\widetilde{\boldsymbol{\psi}}_{\left.\right|_{\Omega^{\prime}}}=\widetilde{\boldsymbol{w}}_{\left.\right|_{\Omega^{\prime}}}-\nabla \Phi^{\prime}=\boldsymbol{w}^{\prime}-\nabla \Phi^{\prime}=\mathbf{0} .
$$

As a consequence, denoting by $\boldsymbol{\psi}$ the restriction of $\widetilde{\boldsymbol{\psi}}$ to $\Omega$, then $\boldsymbol{\psi}$ belongs to $\stackrel{\circ}{W}_{k}^{2}(\Omega)$. Furthermore, in view of (2.19), we have

$$
\operatorname{curl} \widetilde{\boldsymbol{\psi}}=\operatorname{curl} \widetilde{\boldsymbol{w}}=\widetilde{\boldsymbol{u}} \text { in } \mathbb{R}^{3},
$$

which implies that

$$
\boldsymbol{u}=\operatorname{curl} \boldsymbol{\psi} \text { in } \Omega .
$$


The second lemma is a result on a variant of de Rham's theorem.

Lemma 2.7. Suppose that $\Omega$ is of class $\mathscr{C}^{1,1}$ and let $k \in \mathbb{Z}$. Assume that $\boldsymbol{f}$ belongs to $W_{k}^{-1}(\Omega)$ and satisfies

$$
\text { curlf }=\mathbf{0} \text { in } \Omega \text {. }
$$

Then, there exists $p \in W_{k}^{0}(\Omega)$ such that $\boldsymbol{f}=\nabla p$.

Proof. Let us introduce the space

$$
\mathscr{D}_{\sigma}(\Omega)=\{\boldsymbol{\varphi} \in \mathscr{D}(\Omega), \operatorname{div} \boldsymbol{\varphi}=0\} .
$$

It is clear that if $\boldsymbol{\varphi}$ is in $\mathscr{D}_{\sigma}(\Omega)$, then it also belongs to $\mathscr{W}_{-k}^{1}(\Omega)$. Thus, according to Lemma 2.6, there exists $\boldsymbol{\psi} \in \stackrel{\circ}{W}_{-k}^{2}(\Omega)$ such that $\boldsymbol{\varphi}=\operatorname{curl} \boldsymbol{\psi}$. We can now write

$$
\langle\boldsymbol{f}, \boldsymbol{\varphi}\rangle_{W_{k}^{-1}(\Omega) \times \stackrel{W}{L}_{-k}^{1}(\Omega)}=\langle\boldsymbol{f}, \operatorname{curl} \boldsymbol{\psi}\rangle_{W_{k}^{-1}(\Omega) \times \stackrel{\circ}{W}_{-k}^{1}(\Omega)}=\langle\operatorname{curl} \boldsymbol{f}, \boldsymbol{\psi}\rangle_{W_{k}^{-2}(\Omega) \times \stackrel{\circ}{W}_{-k}^{2}(\Omega)}=0 .
$$

Hence, thanks to de Rham's theorem, there exists $q \in \mathscr{D}^{\prime}(\Omega)$ such that $\boldsymbol{f}=\nabla q$. It follows from [29, Theorem 2.7] that there exists a constant c such that $p=q+c \in W_{k}^{0}(\Omega)$ which ends the proof.

Let us now introduce the spaces

$$
W_{k}^{0}(\operatorname{sym} ; \Omega)=\left\{\boldsymbol{e}=\left(e_{i j}\right) \in W_{k}^{0}(\Omega), e_{i j}=e_{j i} \text { in } \Omega\right\}
$$

and

$$
\mathscr{K}(\Omega)=\left\{\boldsymbol{v} \in \mathscr{P}_{1}, \boldsymbol{v}(\boldsymbol{x})=\boldsymbol{a}+\boldsymbol{b} \times \boldsymbol{x}, \boldsymbol{a}, \boldsymbol{b} \in \mathbb{R}^{3}\right\} .
$$

The theorem below gives conditions that have to satisfy symmetric matrix fields to be identified as the rate-of-strain tensor of a vector field.

Theorem 2.8. Suppose that $\Omega$ is of class $\mathscr{C}^{1,1}$ and let $k \in \mathbb{Z}$. Assume that e belongs to $W_{k}^{0}(\operatorname{sym} ; \Omega)$ and satisfies the so-called Saint-Venant compatibility conditions

$$
\forall i, j, k, \ell=1,2,3, \quad \mathscr{R}_{i j k \ell}(\boldsymbol{e})=\partial_{\ell j} e_{i k}+\partial_{k i} e_{j \ell}-\partial_{\ell i} e_{j k}-\partial_{k j} e_{i \ell}=0 .
$$

Then, there exists $\boldsymbol{v} \in W_{k}^{1}(\Omega)$ such that $\boldsymbol{e}=\boldsymbol{D}(\boldsymbol{v})$. The vector field $\boldsymbol{v}$ is unique if $k \geq 0$ and unique up to $a$ an element of $\mathscr{K}(\Omega)$ if $k \leq-1$.

Proof.

- UniQueness : If $\boldsymbol{v}$ belongs to $\mathscr{D}^{\prime}(\Omega)$ and satisfies $\mathbf{D}(\boldsymbol{v})=\mathbf{0}$, then $\boldsymbol{v}$ belongs to $\mathscr{K}(\Omega)$ (see for instance [20]). If in addition $\boldsymbol{v}$ belongs to $W_{k}^{1}(\Omega)$ with $k \geq 0$, then necessarily $\boldsymbol{v}=\mathbf{0}$ because in this case, there are no polynomials in the space $W_{k}^{1}(\Omega)$ (see Proposition 2.1). Observe that if $k=-1$, then the space $\mathscr{K}(\Omega)$ is reduced to $\mathscr{P}_{0}$.

- Existence : Let $\boldsymbol{e}$ be in $W_{k}^{0}(\operatorname{sym} ; \Omega)$ and for any $i, j=1,2,3$, let $\boldsymbol{f}_{i j}$ be defined by

$$
f_{i j k}=\partial_{j} e_{i k}-\partial_{i} e_{j k}, \quad \forall k=1,2,3 .
$$

Then clearly $\boldsymbol{f}_{i j}$ belongs to $W_{k}^{-1}(\Omega)$ and one can observe that we have curl $\boldsymbol{f}_{i j}=\mathbf{0}$ in $\Omega$. Thanks to Lemma 2.7, there exists $p_{i j} \in W_{k}^{0}(\Omega)$ such that $\boldsymbol{f}_{i j}=\nabla p_{i j}$. Observe also that $\nabla\left(p_{i j}+p_{j i}\right)=\mathbf{0}$ with $p_{i j}+p_{j i} \in W_{k}^{0}(\Omega)$. We deduce that, if $k \geq-1, p_{i j}+p_{j i}=0$ and if $k \leq-2$, there exists a unique constant $c_{i j}$ such that $p_{i j}+p_{j i}=c_{i j}$ and so we can choose the functions $p_{i j}$ such that $p_{i j}+p_{j i}=0$. Set now $q_{i j}=e_{i j}+p_{i j} \in W_{k}^{0}(\Omega) \subset W_{k-1}^{-1}(\Omega)$. One can verify that curl $\boldsymbol{q}_{i}=\mathbf{0}$. Therefore, using again Lemma 2.7, there exists $v_{i}$ that belongs to $W_{k-1}^{0}(\Omega)$ such that $\boldsymbol{q}_{i}=\nabla v_{i}$. This shows that $\nabla v_{i}$ belongs to $W_{k}^{0}(\Omega)$. Consequently $v_{i}$ belongs to $W_{k}^{1}(\Omega)$. Besides, since $p_{i j}+p_{j i}=0$, we have

$$
\frac{1}{2}\left(\partial_{j} v_{i}+\partial_{i} v_{j}\right)=\frac{1}{2}\left(q_{i j}+q_{j i}\right)=e_{i j}+\frac{1}{2}\left(p_{i j}+p_{i j}\right)=e_{i j},
$$

which shows that $\boldsymbol{e}=\mathbf{D}(\boldsymbol{v})$. 
We are now in a position to state variant weighted Korn's inequalities.

Theorem 2.9. Suppose that $\Omega$ is of class $\mathscr{C}^{1,1}$ and let $k \geq 0$ be an integer. Then, there exists $C>0$ such that

$$
\forall \boldsymbol{v} \in W_{k}^{1}(\Omega), \quad\|\boldsymbol{v}\|_{W_{k}^{1}(\Omega)} \leq C\|\boldsymbol{D}(\boldsymbol{v})\|_{W_{k}^{0}(\Omega)} .
$$

Proof. Let us introduce the space

$$
\mathscr{E}_{k}(\Omega)=\left\{\boldsymbol{e} \in W_{k}^{0}(\mathrm{sym} ; \Omega), \mathscr{R}_{i j k \ell}(\boldsymbol{e})=0\right\} .
$$

Theorem 2.8 allows to introduce the following linear mapping

$$
\mathscr{F}: \mathscr{E}_{k}(\Omega) \mapsto W_{k}^{1}(\Omega)
$$

defined for each $\boldsymbol{e} \in \mathscr{E}_{k}(\Omega)$ by $\mathscr{F}(\boldsymbol{e})=\boldsymbol{v}$ the unique element of $W_{k}^{1}(\Omega)$ that satisfies $\boldsymbol{e}=\mathbf{D}(\boldsymbol{v})$.

The mapping $\mathscr{F}$ is injective since $\mathscr{F}(\boldsymbol{e})=\mathbf{0}$ implies that $\boldsymbol{v}=\mathbf{0}$ which implies that $\boldsymbol{e}=\mathbf{D}(\mathbf{0})=\mathbf{0}$. Next, for any $\boldsymbol{v} \in W_{k}^{1}(\Omega)$, then clearly $\mathbf{D}(\boldsymbol{v})$ belongs to $\mathscr{E}_{k}(\Omega)$ and this implies that the mapping $\mathscr{F}$ is onto. Finally the inverse mapping $\mathscr{F}^{-1}: W_{k}^{1}(\Omega) \mapsto \mathscr{E}_{k}(\Omega)$ defined for each $\boldsymbol{v} \in W_{k}^{1}(\Omega)$ by $\mathscr{F}^{-1}(\boldsymbol{v})=\mathbf{D}(\boldsymbol{v})$, is clearly continuous. Hence from the closed Range Theorem of Banach, the mapping $\mathscr{F}$ is an isomorphism. As a consequence, there exists a constant $C>0$ such that

$$
\forall \boldsymbol{e} \in \mathscr{E}_{k}(\Omega), \quad\|\mathscr{F}(\boldsymbol{e})\|_{W_{k}^{1}(\Omega)} \leq C\|\boldsymbol{e}\|_{W_{k}^{0}(\Omega)},
$$

which imples (2.23).

When $k \leq-1$, we have similar result in a quotient space because the uniqueness of the vector field $v$ in Theorem 2.8 is up to an element of $\mathscr{K}(\Omega)$.

Theorem 2.10. Suppose that $\Omega$ is of class $\mathscr{C}^{1,1}$ and let $k \leq-1$ be an integer. Then, there exists $C>0$ such that

$$
\forall \boldsymbol{v} \in W_{k}^{1}(\Omega), \quad \inf _{\boldsymbol{q} \in \mathscr{K}(\Omega)}\|\boldsymbol{v}+\boldsymbol{q}\|_{W_{k}^{1}(\Omega)} \leq C\|\boldsymbol{D}(\boldsymbol{v})\|_{W_{k}^{0}(\Omega)}
$$

\section{The exterior Stokes problem with Navier boundary conditions}

In this section we consider the Stokes problem with Navier slip boundary conditions:

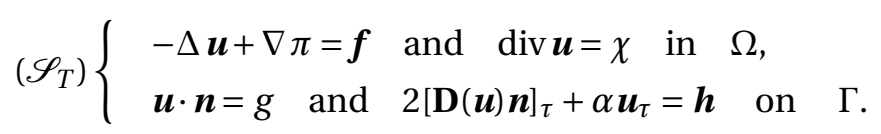

Our aim is to investigate the existence and the uniqueness of a solution $(\boldsymbol{u}, \pi)$ to $\left(\mathscr{S}_{T}\right)$ that belongs at least to $W_{k}^{1}(\Omega) \times W_{k}^{0}(\Omega)$. We begin by looking for the variational solution which corresponds to the case $k=0$. From now on, we assume that $\alpha$ is a positive function that belongs to $L^{\infty}(\Gamma)$.

\subsection{Variational solution}

As usual the existence and the uniqueness of a variational solution to $\left(\mathscr{S}_{T}\right)$ rely on the Lax-Milgram theorem. The proposition below gives a variational formulation for $\left(\mathscr{S}_{T}\right)$.

Proposition 3.1. Suppose that $\Omega$ is of class $\mathscr{C}^{1,1}$. Assume that $\chi=g=0$, let $\boldsymbol{f} \in H_{1}^{-1}(\operatorname{div} ; \Omega)$ and $\boldsymbol{h} \in$ $H^{-1 / 2}(\Gamma)$ such that

$$
\boldsymbol{h} \cdot \boldsymbol{n}=0 \quad \text { on } \quad \Gamma .
$$


Then, finding $(\boldsymbol{u}, \pi) \in W_{0}^{1}(\Omega) \times L^{2}(\Omega)$ satisfying $\left(\mathscr{S}_{T}\right)$ in the sense of distributions, is equivalent to the problem:

$$
\left\{\begin{array}{l}
\text { find } \boldsymbol{u} \in V_{\sigma, T}(\Omega) \text { such that, } \\
\quad 2 \int_{\Omega} \boldsymbol{D}(\boldsymbol{u}): \mathbf{D}(\boldsymbol{\varphi}) d \boldsymbol{x}+\int_{\Gamma} \alpha(\boldsymbol{x}) \boldsymbol{u}_{\tau} \boldsymbol{\varphi} d \sigma=\langle\boldsymbol{f}, \boldsymbol{\varphi}\rangle_{\Omega}+\langle\boldsymbol{h}, \boldsymbol{\varphi}\rangle_{\Gamma}, \quad \forall \boldsymbol{\varphi} \in V_{\sigma, T}(\Omega),
\end{array}\right.
$$

where we recall that the space $V_{\sigma, T}(\Omega)$ is defined in (2.12).

Proof. Let $(\boldsymbol{u}, \pi) \in W_{0}^{1}(\Omega) \times L^{2}(\Omega)$ be a solution of $\left(\mathscr{S}_{T}\right)$. Thanks to the characterization given in Proposition $2.2,-\Delta \boldsymbol{u}=\boldsymbol{f}-\nabla \pi$ belongs to $H_{1}^{-1}(\operatorname{div} ; \Omega)$ and thus, $\boldsymbol{u}$ is in the space $E(\Omega)$ defined in (2.13). Then we can write

$$
\forall \boldsymbol{\varphi} \in V_{\sigma, T}(\Omega) \subset \stackrel{\circ}{H}_{-1}(\operatorname{div} ; \Omega), \quad\langle-\Delta \boldsymbol{u}+\nabla \pi, \boldsymbol{\varphi}\rangle_{\Omega}=\langle-\Delta \boldsymbol{u}, \boldsymbol{\varphi}\rangle_{\Omega}+\langle\nabla \pi, \boldsymbol{\varphi}\rangle_{\Omega}=\langle\boldsymbol{f}, \boldsymbol{\varphi}\rangle_{\Omega},
$$

where $\langle\cdot, \cdot\rangle_{\Omega}$ denotes the dualities between $H_{1}^{-1}(\operatorname{div} ; \Omega)$ and $\stackrel{\circ}{H}_{-1}(\operatorname{div} ; \Omega)$. Due to the density of $\mathscr{D}(\Omega)$ in $\stackrel{\circ}{H}_{-1}(\operatorname{div} ; \Omega)$, we have

$$
\langle\nabla \pi, \boldsymbol{\varphi}\rangle_{\Omega}=-\int_{\Omega} \pi \operatorname{div} \boldsymbol{\varphi} d \boldsymbol{x}, \quad \forall \boldsymbol{\varphi} \in \stackrel{\circ}{H}_{-1}(\operatorname{div} ; \Omega)
$$

Particularly

$$
\langle\nabla \pi, \boldsymbol{\varphi}\rangle_{\Omega}=0 \quad \text { if } \boldsymbol{\varphi} \in V_{\sigma, T}(\Omega) .
$$

Now using the Green formula (2.14) in (3.3), we deduce that every solution of $\left(\mathscr{S}_{T}\right)$ also solves (3.2).

Conversely, let $\boldsymbol{u} \in V_{\sigma, T}(\Omega)$ be a solution of (3.2). Then for all $\boldsymbol{\varphi} \in \mathscr{D}_{\sigma}(\Omega)$ (defined in (2.21)), we have on the one hand

$$
2 \int_{\Omega} \mathbf{D}(\boldsymbol{u}): \mathbf{D}(\boldsymbol{\varphi}) d \boldsymbol{x}=\langle\boldsymbol{f}, \boldsymbol{\varphi}\rangle_{\mathscr{D}^{\prime}(\Omega) \times \mathscr{D}(\Omega)}
$$

and on the other hand

$$
2 \int_{\Omega} \mathbf{D}(\boldsymbol{u}): \mathbf{D}(\boldsymbol{\varphi}) d \boldsymbol{x}=-\langle\Delta \boldsymbol{u}, \boldsymbol{\varphi}\rangle_{\mathscr{D}^{\prime}(\Omega) \times \mathscr{D}(\Omega)} .
$$

As a consequence, we obtain

$$
\langle-\Delta \boldsymbol{u}-\boldsymbol{f}, \boldsymbol{\varphi}\rangle_{\mathscr{D}^{\prime}(\Omega) \times \mathscr{D}(\Omega)}=0, \quad \forall \boldsymbol{\varphi} \in \mathscr{D}_{\sigma}(\Omega) .
$$

Hence, by de Rham's theorem, there exists $q \in \mathscr{D}^{\prime}(\Omega)$ such that

$$
-\Delta \boldsymbol{u}-\boldsymbol{f}=\nabla q \text { in } \Omega .
$$

Thanks to (2.8), we have $\boldsymbol{f} \in H_{1}^{-1}(\operatorname{div} ; \Omega) \subset W_{0}^{-1}(\Omega)$ and thus $-\Delta \boldsymbol{u}-\boldsymbol{f} \in W_{0}^{-1}(\Omega)$. It follows from [29, Theorem 2.7], that there exists a constant $c$ such that $\pi=q+c \in L^{2}(\Omega)$. Because $\boldsymbol{u}$ belongs to $V_{\sigma, T}(\Omega)$, we have $\operatorname{div} \boldsymbol{u}=0$ in $\Omega$ and $\boldsymbol{u} \cdot \boldsymbol{n}=0$ on $\Gamma$.

It remains to prove that the second boundary condition $2[\mathbf{D}(\boldsymbol{u}) \boldsymbol{n}]_{\tau}+\alpha \boldsymbol{u}_{\tau}=\boldsymbol{h}$ on $\Gamma$ holds. To that end, let us multiply (3.4) by $\boldsymbol{\varphi} \in V_{\sigma, T}(\Omega) \subset \stackrel{\circ}{H}_{-1}(\operatorname{div} ; \Omega)$. This yields

$$
\langle-\Delta \boldsymbol{u}+\nabla \pi, \boldsymbol{\varphi}\rangle_{\Omega}=\langle\boldsymbol{f}, \boldsymbol{\varphi}\rangle_{\Omega} .
$$

Observe now that $\boldsymbol{u}$ also belongs to $E(\Omega)$. Hence, using the Green formula (2.14), we obtain:

$$
2 \int_{\Omega} \mathbf{D}(\boldsymbol{u}): \mathbf{D}(\boldsymbol{\varphi}) d \boldsymbol{x}-\left\langle 2[\mathbf{D}(\boldsymbol{u}) \boldsymbol{n}]_{\tau}, \boldsymbol{\varphi}\right\rangle_{\Gamma}=\langle\boldsymbol{f}, \boldsymbol{\varphi}\rangle_{\Omega} .
$$


Therefore, from (3.2) and (3.5) we deduce that

$$
\forall \boldsymbol{\varphi} \in V_{\sigma, T}(\Omega), \quad\left\langle 2[\mathbf{D}(\boldsymbol{u}) \boldsymbol{n}]_{\tau}+\alpha \boldsymbol{u}_{\tau}, \boldsymbol{\varphi}\right\rangle_{\Gamma}=\langle\boldsymbol{h}, \boldsymbol{\varphi}\rangle_{\Gamma} .
$$

Let now $\boldsymbol{\mu}$ be any element of the space $H^{1 / 2}(\Gamma)$. So, there exists $\boldsymbol{\varphi} \in W_{0}^{1}(\Omega)$ such as $\operatorname{div} \boldsymbol{\varphi}=0$ in $\Omega$ and $\boldsymbol{\varphi}=\boldsymbol{\mu}_{\boldsymbol{\tau}}$ on $\Gamma$. It is clear that $\boldsymbol{\varphi} \in V_{\sigma, T}(\Omega)$ and

$$
\begin{aligned}
\left\langle 2[\mathbf{D}(\boldsymbol{u}) \boldsymbol{n}]_{\tau}+\alpha \boldsymbol{u}_{\tau}-\boldsymbol{h}, \boldsymbol{\mu}\right\rangle_{\Gamma} & =\left\langle 2[\mathbf{D}(\boldsymbol{u}) \boldsymbol{n}]_{\tau}+\alpha \boldsymbol{u}_{\tau}-\boldsymbol{h}, \boldsymbol{\mu}_{\tau}\right\rangle_{\Gamma} \\
& =\left\langle 2[\mathbf{D}(\boldsymbol{u}) \boldsymbol{n}]_{\tau}+\alpha \boldsymbol{u}_{\tau}-\boldsymbol{h}, \boldsymbol{\varphi}\right\rangle_{\Gamma} \\
& =0 .
\end{aligned}
$$

This implies that

$$
2[\mathbf{D}(\boldsymbol{u}) \boldsymbol{n}]_{\tau}+\alpha \boldsymbol{u}_{\tau}=\boldsymbol{h} \text { on } \Gamma
$$

Proposition 3.2. Suppose that $\Omega$ is of class $\mathscr{C}^{1,1}$. Assume that $\chi=g=0$ and let $\boldsymbol{f} \in H_{1}^{-1}(\operatorname{div}, \Omega)$ and $\boldsymbol{h} \in$ $H^{-1 / 2}(\Gamma)$ satisfying (3.1). Then, the Stokes problem $\left(\mathscr{S}_{T}\right)$ has a unique solution $(\boldsymbol{u}, \pi) \in W_{0}^{1}(\Omega) \times L^{2}(\Omega)$. In addition, we have the following estimate:

$$
\|\boldsymbol{u}\|_{W_{0}^{1}(\Omega)}+\|\pi\|_{L^{2}(\Omega)} \leqslant C\left(\|\boldsymbol{f}\|_{H_{1}^{-1}(\operatorname{div} ; \Omega)}+\|\boldsymbol{h}\|_{H^{-1 / 2}(\Gamma)}\right) .
$$

Proof. Due to the equivalence between problem $\left(\mathscr{S}_{T}\right)$ and the variational formulation (3.2), it is enough to solve (3.2). This is done by the use of Lax-Milgram's theorem. Consider the bilinear form

$$
\mathscr{A}: V_{\sigma, T}(\Omega) \times V_{\sigma, T}(\Omega) \rightarrow \mathbb{R}
$$

defined by

$$
\mathscr{A}(\boldsymbol{u}, \boldsymbol{v})=\int_{\Omega} \mathbf{D}(\boldsymbol{u}): \mathbf{D}(\boldsymbol{v}) d \boldsymbol{x}+\int_{\Gamma} \alpha(\boldsymbol{x}) \boldsymbol{u}_{\tau} \boldsymbol{v}_{\tau} d \boldsymbol{x} .
$$

The mapping $\mathscr{A}(\cdot, \cdot)$ is clearly continuous and thanks to the Korn's inequality (2.23), it is also coercive in $V_{\sigma, T}(\Omega)$. Moreover, the linear form $\ell: V_{\sigma, T}(\Omega) \rightarrow \mathbb{R}$, defined by

$$
\ell(\boldsymbol{v})=\langle\boldsymbol{f}, \boldsymbol{u}\rangle_{\Omega}+\langle\boldsymbol{h}, \boldsymbol{v}\rangle_{\Gamma},
$$

is continuous on $V_{\sigma, T}(\Omega)$. Hence, using Lax-Milgram's theorem, we deduce that problem (3.2) has a unique solution $\boldsymbol{u}$ in $V_{\sigma, T}(\Omega)$.

Let us now prove estimate (3.6). On the one hand, using the variational problem and Korn's inequality (2.23), we obtain

$$
\|\boldsymbol{u}\|_{W_{0}^{1}(\Omega)} \leq C\left(\|\boldsymbol{f}\|_{H_{1}^{-1}(\mathrm{div} ; \Omega)}+\|\boldsymbol{h}\|_{H^{-1 / 2}(\Gamma)}\right) .
$$

On the other hand, we have

$$
\begin{aligned}
\|\nabla \pi\|_{W_{0}^{-1}(\Omega)} & \leq\|\boldsymbol{f}\|_{H_{1}^{-1}(\mathrm{div} ; \Omega)}+\|\Delta \boldsymbol{u}\|_{W_{0}^{-1}(\Omega)} \\
& \leq C\left(\|\boldsymbol{f}\|_{H_{1}^{-1}(\mathrm{div} ; \Omega)}+\|\boldsymbol{u}\|_{W_{0}^{1}(\Omega)}\right) \\
& \leq C\left(\|\boldsymbol{f}\|_{H_{1}^{-1}(\operatorname{div} ; \Omega)}+\|\boldsymbol{h}\|_{H^{-1 / 2}(\Gamma)}\right) .
\end{aligned}
$$

Combining this estimate, (3.7) and the fact that (see [32, Corollary 3.3])

$$
\|\pi\|_{L^{2}(\Omega)} \leq C\|\nabla \pi\|_{W_{0}^{-1}(\Omega)}
$$

allows to obtain (3.6). 
We are now in a position to prove the existence and uniqueness of a variational solution to the complete Stokes problem $\left(\mathscr{S}_{T}\right)$.

Theorem 3.3. Suppose that $\Omega$ is of class $\mathscr{C}^{1,1}$. Assume that $f \in H_{1}^{-1}(\operatorname{div} ; \Omega), \chi \in L^{2}(\Omega), g \in H^{1 / 2}(\Gamma)$ and $\boldsymbol{h} \in H^{-1 / 2}(\Gamma)$ satisfies the compatibility condition (3.1). Then, the Stokes problem $\left(\mathscr{S}_{T}\right)$ has a unique solution $(\boldsymbol{u}, \pi) \in W_{0}^{1}(\Omega) \times L^{2}(\Omega)$. Moreover, the following estimate holds:

$$
\|\boldsymbol{u}\|_{W_{0}^{1}(\Omega)}+\|\pi\|_{L^{2}(\Omega)} \leq C\left(\|\boldsymbol{f}\|_{H_{1}^{-1}(\operatorname{div}, \Omega)}+\|\chi\|_{L^{2}(\Omega)}+\|g\|_{H^{1 / 2}(\Gamma)}+\|\boldsymbol{h}\|_{H^{-1 / 2}(\Gamma)}\right) .
$$

Proof. Let us first solve the below exterior Neumann problem:

$$
\Delta \theta=\chi \quad \text { in } \Omega \text { and } \frac{\partial \theta}{\partial \boldsymbol{n}}=g \text { on } \Gamma .
$$

Thanks to [33] (see also [29, Theorem 3.9]), this problem has a unique solution $\theta \in W_{0}^{2}(\Omega) / \mathbb{R}$ satisfying the estimate:

$$
\|\theta\|_{W_{0}^{2}(\Omega) / \mathbb{R}} \leqslant C\left(\|\chi\|_{L^{2}(\Omega)}+\|g\|_{H^{1 / 2}(\Gamma)}\right) .
$$

Next we look for a pair $(\boldsymbol{z}, \pi) \in W_{0}^{1}(\Omega) \times L^{2}(\Omega)$ satisfying

$$
\left\{\begin{array}{l}
-\Delta \boldsymbol{z}+\nabla \pi=\boldsymbol{f}+\nabla \chi \text { and } \operatorname{div} \boldsymbol{z}=0 \text { in } \Omega, \\
\boldsymbol{z} \cdot \boldsymbol{n}=0 \text { and } 2[\mathbf{D}(\boldsymbol{z}) \boldsymbol{n}]_{\tau}+\alpha \boldsymbol{z}_{\tau}=\boldsymbol{h}^{\prime} \text { on } \Gamma,
\end{array}\right.
$$

where $\boldsymbol{h}^{\prime}=\boldsymbol{h}-2[\mathbf{D}(\nabla \theta) \boldsymbol{n}]_{\tau}-\alpha(\nabla \theta)_{\tau}$.

Observe that in view of the assumption on $\boldsymbol{f}$ and Proposition 2.2, $\boldsymbol{f}+\nabla \chi$ belongs to $H_{1}^{-1}(\operatorname{div} ; \Omega)$. Besides, as $\theta$ belongs to $W_{0}^{2}(\Omega)$, then $\nabla \theta$ belongs to $W_{0}^{1}(\Omega)$ (see (2.1)). As a consequence, $\boldsymbol{h}^{\prime}$ belongs to $H^{-1 / 2}(\Gamma)$ and satisfies

$$
\left\|\boldsymbol{h}^{\prime}\right\|_{H^{-1 / 2}(\Gamma)} \leq C\left(\|\boldsymbol{h}\|_{H^{-1 / 2}(\Gamma)}+\|\theta\|_{W_{0}^{2}(\Omega) / \mathbb{R}}\right)
$$

and clearly satisfies $\boldsymbol{h}^{\boldsymbol{\prime}} \cdot \boldsymbol{n}=0$ on $\Gamma$. According to Proposition 3.2, problem (3.10) has a unique solution $(\boldsymbol{z}, \pi) \in W_{0}^{1}(\Omega) \times L^{2}(\Omega)$ satisfying

$$
\|\boldsymbol{z}\|_{W_{0}^{1}(\Omega)}+\|\pi\|_{L^{2}(\Omega)} \leq C\left(\|\boldsymbol{f}\|_{H_{1}^{-1}(\operatorname{div} ; \Omega)}+\|\chi\|_{L^{2}(\Omega)}+\left\|\boldsymbol{h}^{\prime}\right\|_{H^{-1 / 2}(\Omega)}\right) .
$$

Thus setting $\boldsymbol{u}=\boldsymbol{z}+\nabla \theta$, the pair $(\boldsymbol{u}, \pi) \in\left(W_{0}^{1}(\Omega) \times L^{2}(\Omega)\right)$ is the unique solution of $\left(\mathscr{S}_{T}\right)$ and estimate (3.8) follows from (3.11) and (3.12).

Remark 3.4. Let us mention that due to Proposition 2.2, the statement of Theorem 3.3 remains valid if we assume that fbelongs to $W_{1}^{0}(\Omega)$. This space is much more convenient for applications and for the numerical approximation of the Stokes problem $\left(\mathscr{S}_{T}\right)$.

\subsection{Strong solutions}

In this part, we investigate the well-posedness of strong solutions in $W_{k+1}^{2}(\Omega) \times W_{k+1}^{1}(\Omega)$ with $k \in \mathbb{Z}$. In the behavior at infinity point of view, we are looking for solutions $(\boldsymbol{u}, \pi)$ that grow or decay weaker at infinity $(k \leq 0)$ than the variational solution or decay faster at infinity $(k>0)$ than this latter solution. In order to deal with the uniqueness issues, we first need to characterize the kernel of problem $\left(\mathscr{S}_{T}\right)$.

For $k \in \mathbb{Z}$, we introduce:

$$
\mathscr{N}_{k}(\Omega)=\left\{(\boldsymbol{u}, \pi) \in W_{k}^{1}(\Omega) \times W_{k}^{0}(\Omega) ;-\Delta \boldsymbol{u}+\nabla \pi=0, \operatorname{div} \boldsymbol{u}=0 \text { in } \Omega \text { and } \boldsymbol{u} \cdot \boldsymbol{n}=0,2[\mathbf{D}(\boldsymbol{u}) \boldsymbol{n}]_{\tau}+\alpha \boldsymbol{u}_{\tau}=0 \text { on } \Gamma\right\} .
$$

The characterization of the kernel $\mathscr{N}_{k}(\Omega)$ is given by the following proposition:

Proposition 3.5. Suppose that $\Omega$ is of class $\mathscr{C}^{1,1}$ and assume $k \in \mathbb{Z}$. 
- If $k \geq 0$, then $\mathscr{N}_{k}(\Omega)=\{(\mathbf{0}, 0)\}$.

- If $k<0$, then $\mathscr{N}_{k}(\Omega)=\left\{(\boldsymbol{v}-\boldsymbol{\lambda}, \theta-\mu) ; \quad(\boldsymbol{\lambda}, \mu) \in N_{-k-1}\right\}$, where $(\boldsymbol{v}, \theta) \in W_{0}^{1}(\Omega) \times L^{2}(\Omega)$ is the unique solution of the following problem:

$$
\left\{\begin{array}{l}
-\Delta \boldsymbol{v}+\nabla \theta=\mathbf{0} \text { and } \operatorname{div} \boldsymbol{v}=0 \quad \text { in } \Omega, \\
\boldsymbol{v} \cdot \boldsymbol{n}=\boldsymbol{\lambda} \cdot \boldsymbol{n} \text { and } 2[\boldsymbol{D}(\boldsymbol{v}) \boldsymbol{n}]_{\tau}+\alpha \boldsymbol{v}_{\tau}=2[\boldsymbol{D}(\boldsymbol{\lambda}) \boldsymbol{n}]_{\tau}+\alpha \boldsymbol{\lambda}_{\tau} \text { on } \Gamma .
\end{array}\right.
$$

Moreover, the space $\mathscr{N}_{k}(\Omega)$ is a finite-dimensional space and $\operatorname{dim} \mathscr{N}_{k}(\Omega)=\operatorname{dim} N_{-k-1}=\operatorname{dim} \mathscr{P}_{-k-1} \times \mathscr{P}_{-k-2}^{\Delta}$.

Proof.

- If $k \geq 0$, because $W_{k}^{1}(\Omega) \times W_{k}^{0}(\Omega) \hookrightarrow W_{0}^{1}(\Omega) \times L^{2}(\Omega)$, we have $\mathscr{N}_{k}(\Omega) \subset \mathscr{N}_{0}(\Omega)$. Now thanks to Theorem 3.2, we have $\mathscr{N}_{0}(\Omega)=\{(\mathbf{0}, 0)\}$ which ends the proof for this case.

- If $k<0$, then let us assume that $(\boldsymbol{u}, \pi)$ belongs to $\mathscr{N}_{k}(\Omega)$. The pair $(\boldsymbol{u}, \pi)$ has an extension $(\widetilde{\boldsymbol{u}}, \widetilde{\pi})$ that belongs to $W_{k}^{1}\left(\mathbb{R}^{3}\right) \times W_{k}^{0}\left(\mathbb{R}^{3}\right)$. Set now

$$
\boldsymbol{F}=-\Delta \widetilde{\boldsymbol{u}}+\nabla \widetilde{\pi} \text { and } e=\operatorname{div} \widetilde{\boldsymbol{u}} .
$$

Then the pair $(\boldsymbol{F}, e)$ belongs to $W_{k}^{-1}\left(\mathbb{R}^{3}\right) \times W_{k}^{0}\left(\mathbb{R}^{3}\right)$ and has a compact support. Therefore $(\boldsymbol{F}, e)$ also belongs to $W_{0}^{-1}\left(\mathbb{R}^{3}\right) \times L^{2}\left(\mathbb{R}^{3}\right)$. It follows from Theorem 2.4 , that there exists a solution $(\boldsymbol{v}, \theta) \in W_{0}^{1}\left(\mathbb{R}^{3}\right) \times L^{2}\left(\mathbb{R}^{3}\right)$ such that

$$
-\Delta \boldsymbol{v}+\nabla \theta=-\Delta \widetilde{\boldsymbol{u}}+\nabla \widetilde{\pi} \text { and } \operatorname{div} \boldsymbol{v}=\operatorname{div} \widetilde{\boldsymbol{u}} \text { in } \mathbb{R}^{3} .
$$

The fact that $k<0$ implies that $(\boldsymbol{v}-\widetilde{\boldsymbol{u}}, \theta-\widetilde{\pi})$ also belongs to $W_{k}^{1}\left(\mathbb{R}^{3}\right) \times W_{k}^{0}\left(\mathbb{R}^{3}\right)$. It follows from Proposition 2.3 that $\boldsymbol{v}=\widetilde{\boldsymbol{u}}+\boldsymbol{\lambda}$ and $\theta=\widetilde{\boldsymbol{\pi}}+\mu$, where $(\boldsymbol{\lambda}, \mu) \in N_{-k-1}$. We deduce that the restriction of $\boldsymbol{v}$ and $\theta$ to $\Omega$ satisfy (3.13).

Let us now prove that the kernel $\mathscr{N}_{k}(\Omega)$ has a finite dimension. Thanks to Theorem 3.3, we can introduce the linear mapping

$$
\mathscr{T}: N_{-k-1} \mapsto \mathscr{N}_{k}(\Omega)
$$

defined for each $(\boldsymbol{\lambda}, \mu) \in N_{-k-1}$, by $\mathscr{T}(\boldsymbol{\lambda}, \mu)=(\boldsymbol{\nu}(\boldsymbol{\lambda})-\boldsymbol{\lambda}, \theta(\boldsymbol{\lambda})-\mu)$, where $\left(\boldsymbol{\nu}(\boldsymbol{\lambda}, \theta(\boldsymbol{\lambda})) \in W_{0}^{1}(\Omega) \times L^{2}(\Omega)\right.$ is the unique solution of (3.13).

Using the first part of the proof, this mapping is clearly onto. It is also injective, since $\mathscr{T}(\boldsymbol{\lambda}, \mu)=\{(\mathbf{0}, 0)\}$, implies that the pair $(\boldsymbol{v}(\boldsymbol{\lambda}), \theta(\boldsymbol{\lambda})) \in W_{0}^{1}(\Omega) \times L^{2}(\Omega)$ is a pair of polynomials of $\mathscr{P}_{-k-1} \times \mathscr{P}_{-k-2}^{\Delta}$. But the space $W_{0}^{1}(\Omega) \times$ $L^{2}(\Omega)$ does not contain polynomials, which implies that $(\boldsymbol{v}(\boldsymbol{\lambda}), \theta(\boldsymbol{\lambda}))=\{(\mathbf{0}, 0)\}$ which in turn also implies that $(\boldsymbol{\lambda}, \mu)=\{(\mathbf{0}, 0)\}$. Finally, thanks to (3.8), $\mathscr{T}$ is continuous. Hence the operator $\mathscr{T}$ is an isomorphism from $N_{-k-1}$ to $\mathscr{N}_{k}(\Omega)$. Since the dimension of $N_{-k-1}$ is finite and is equal to the dimension of $\mathscr{P}_{-k-1} \times \mathscr{P}_{-k-2}^{\Delta}$, this ends the proof.

We start by looking for strong solutions for the case $k \leq 0$.

Theorem 3.6. Suppose that $\Omega$ is of class $\mathscr{C}^{2,1}$ and let $k \leq 0$ be an integer. Assume that $\boldsymbol{f} \in W_{k+1}^{0}(\Omega), \chi \in W_{k+1}^{1}(\Omega)$, $g \in H^{3 / 2}(\Gamma)$ and $\boldsymbol{h} \in H^{1 / 2}(\Gamma)$ satisfying (3.1). Then the Stokes problem $\left(\mathscr{S}_{T}\right)$ has a solution $(\boldsymbol{u}, \pi) \in W_{k+1}^{2}(\Omega) \times$ $W_{k+1}^{1}(\Omega)$ unique up to an element of $\mathscr{N}_{k}(\Omega)$ and we have:

$$
\inf _{(\boldsymbol{\lambda}, \mu) \in \mathcal{N}_{k}(\Omega)}\left(\|\boldsymbol{u}+\boldsymbol{\lambda}\|_{W_{k+1}^{2}(\Omega)}+\|\pi+\mu\|_{W_{k+1}^{1}(\Omega)}\right) \leqslant C\left(\|\boldsymbol{f}\|_{W_{k+1}^{0}(\Omega)}+\|\chi\|_{W_{k+1}^{1}(\Omega)}+\|g\|_{H^{3 / 2}(\Gamma)}+\|\boldsymbol{h}\|_{H^{1 / 2}(\Gamma)}\right) .
$$

Proof. The uniqueness result follows immediately from Proposition 3.5. The proof of the existence result is made of two steps.

- We first establish the existence of a solution in $W_{k}^{1}(\Omega) \times W_{k}^{0}(\Omega)$.

The main idea is to use the results on the Stokes problem in the whole space $\mathbb{R}^{3}$. To that end, let $\widetilde{f}$ denote the extension by zero of $\boldsymbol{f}$. Then $\widetilde{\boldsymbol{f}}$ belongs to $W_{k+1}^{0}\left(\mathbb{R}^{3}\right)$. Besides, by lifting the trace of $\chi$ on $\Gamma$ to $\Omega^{\prime}$, we have an extension $\tilde{\chi}$ of $\chi$ that belongs to $W_{k+1}^{1}\left(\mathbb{R}^{3}\right)$. Thanks to Theorem 2.4, the following Stokes problem in $\mathbb{R}^{3}$

$$
-\Delta \widetilde{\boldsymbol{u}}+\nabla \widetilde{\boldsymbol{\pi}}=\widetilde{\boldsymbol{f}} \text { and } \operatorname{div} \widetilde{\boldsymbol{u}}=\widetilde{\chi} \text { in } \mathbb{R}^{3},
$$

has a solution $(\widetilde{\boldsymbol{u}}, \widetilde{\pi})$ that belongs to $W_{k+1}^{2}\left(\mathbb{R}^{3}\right) \times W_{k+1}^{0}\left(\mathbb{R}^{3}\right)$. 
Let us now consider the following problem:

$$
\left\{\begin{array}{l}
-\Delta z+\nabla \theta=\mathbf{0} \text { and } \operatorname{div} z=0 \quad \text { in } \Omega \\
\boldsymbol{z} \cdot \boldsymbol{n}=\widetilde{\boldsymbol{u}} \cdot \boldsymbol{n}+g \text { and } 2[\mathbf{D}(z) \boldsymbol{n}]_{\tau}+\alpha z_{\tau}=2[\mathbf{D}(\widetilde{\boldsymbol{u}}) \boldsymbol{n}]_{\tau}+\alpha \widetilde{\boldsymbol{u}}_{\tau}+\boldsymbol{h} \text { on } \Gamma .
\end{array}\right.
$$

The fact that $\widetilde{\boldsymbol{u}} \in W_{k+1}^{2}\left(\mathbb{R}^{3}\right)$ and $\boldsymbol{h} \in H^{1 / 2}(\Gamma)$ imply that $2[\mathbf{D}(\widetilde{\boldsymbol{u}}) \boldsymbol{n}]_{\tau}+\alpha \widetilde{\boldsymbol{u}}_{\tau}+\boldsymbol{h} \in H^{1 / 2}(\Gamma) \hookrightarrow H^{-1 / 2}(\Omega)$. Moreover, from (3.1), we clearly have

$$
\left(2[\mathbf{D}(\boldsymbol{u}) \boldsymbol{n}]_{\tau}+\alpha \boldsymbol{u}_{\tau}+\boldsymbol{h}\right) \cdot \boldsymbol{n}=0 \quad \text { on } \quad \Gamma .
$$

Therefore, thanks to Theorem 3.3, Problem (3.14) has a unique solution $(\boldsymbol{z}, \theta) \in W_{0}^{1}(\Omega) \times L^{2}(\Omega)$. Since $k \leqslant 0$, then the pair $(\boldsymbol{z}, \theta)$ also belongs to $W_{k}^{1}(\Omega) \times W_{k}^{0}(\Omega)$. Setting now $\boldsymbol{u}=\boldsymbol{z}-\widetilde{\boldsymbol{u}}_{\mid \Omega}$ and $\pi=\theta-\widetilde{\pi}_{\mid \Omega}$, then the pair $(\boldsymbol{u}, \pi)$ belongs to $W_{k}^{1}(\Omega) \times W_{k}^{0}(\Omega)$ and satisfies $\left(\mathscr{S}_{T}\right)$.

- We shall now prove that the pair $(\boldsymbol{u}, \pi)$ belongs to $W_{k+1}^{2}(\Omega) \times W_{k+1}^{1}(\Omega)$.

Here we use regularity arguments on the Stokes problem set in bounded domains and in the whole space $\mathbb{R}^{3}$. To that end, let us introduce the following partition of unity:

$$
\begin{aligned}
& \varphi, \psi \in \mathscr{C}^{\infty}\left(\mathbb{R}^{3}\right), \quad 0 \leq \varphi, \psi \leq 1, \quad \varphi+\psi=1 \quad \text { in } \mathbb{R}^{3}, \\
& \varphi=1 \quad \text { in } \quad B_{R}, \quad \operatorname{supp} \varphi \subset B_{R+1} .
\end{aligned}
$$

Let $(\widetilde{\boldsymbol{u}}, \widetilde{\pi}) \in W_{k}^{1}\left(\mathbb{R}^{3}\right) \times W_{k}^{0}\left(\mathbb{R}^{3}\right)$ be an extension of $(\boldsymbol{u}, \pi)$ to the whole space $\mathbb{R}^{3}$. We can write:

$$
\widetilde{\boldsymbol{u}}=\varphi \widetilde{\boldsymbol{u}}+\psi \widetilde{\boldsymbol{u}} \text { and } \widetilde{\pi}=\varphi \widetilde{\pi}+\psi \widetilde{\pi} .
$$

Then it is enough to show that the pairs $(\varphi \widetilde{\boldsymbol{u}}, \varphi \widetilde{\pi})$ and $(\psi \widetilde{\boldsymbol{u}}, \psi \widetilde{\pi})$ belong to $W_{k+1}^{2}\left(\mathbb{R}^{3}\right) \times W_{k+1}^{1}\left(\mathbb{R}^{3}\right)$. To that end, consider first

$$
-\Delta(\psi \widetilde{\boldsymbol{u}})+\nabla(\psi \widetilde{\pi})=\boldsymbol{f}_{1} \text { and } \operatorname{div}(\psi \widetilde{\boldsymbol{u}})=\chi_{1} \quad \text { in } \quad \mathbb{R}^{3},
$$

where

$$
\boldsymbol{f}_{1}=\boldsymbol{f} \psi-(2 \nabla \widetilde{\boldsymbol{u}} \nabla \psi+\boldsymbol{u} \Delta \psi)+\widetilde{\pi} \nabla \psi \quad \text { and } \quad \chi_{1}=\chi \psi+\widetilde{\boldsymbol{u}} \cdot \nabla \psi \text {. }
$$

We easily see that $\boldsymbol{f}_{1}$ and $\chi_{1}$ have the same regularity as $\boldsymbol{f}$ and $\chi$ respectively and so $\left(\boldsymbol{f}_{1}, \chi_{1}\right)$ belongs to $W_{k+1}^{0}\left(\mathbb{R}^{3}\right) \times W_{k+1}^{1}\left(\mathbb{R}^{3}\right)$. Therefore, thanks to Theorem 2.4 , there exists $(\boldsymbol{w}, q) \in W_{k+1}^{2}\left(\mathbb{R}^{3}\right) \times W_{k+1}^{1}\left(\mathbb{R}^{3}\right)$ satisfying

$$
-\Delta \boldsymbol{w}+\nabla q=\boldsymbol{f}_{1} \quad \text { and } \quad \operatorname{div} \boldsymbol{w}=\chi_{1} \text { in } \mathbb{R}^{3} .
$$

Let $\boldsymbol{v}=\psi \widetilde{\boldsymbol{u}}-\boldsymbol{w}$ and $\theta=\psi \widetilde{\pi}-q$, then $(\boldsymbol{v}, \theta)$ belongs to $W_{k}^{1}\left(\mathbb{R}^{3}\right) \times W_{k}^{0}\left(\mathbb{R}^{3}\right)$ and satisfies

$$
-\Delta \boldsymbol{v}+\nabla \theta=\mathbf{0} \text { and } \operatorname{div} \boldsymbol{v}=0 \text { in } \mathbb{R}^{3} \text {. }
$$

It follows from Proposition 2.3 that $(\boldsymbol{v}, \theta) \in N_{-k-1}$. Since $N_{-k-1} \subset W_{k+1}^{2}\left(\mathbb{R}^{3}\right) \times W_{k+1}^{1}\left(\mathbb{R}^{3}\right)$, we deduce that the pair $(\psi \tilde{\boldsymbol{u}}, \psi \tilde{\pi})$ also belongs to $W_{k+1}^{2}\left(\mathbb{R}^{3}\right) \times W_{k+1}^{1}\left(\mathbb{R}^{3}\right)$.

Consider now the system

$$
-\Delta(\varphi \widetilde{\boldsymbol{u}})+\nabla(\varphi \widetilde{\pi})=\boldsymbol{f}_{2} \quad \text { and } \operatorname{div}(\varphi \widetilde{\boldsymbol{u}})=\chi_{2},
$$

where $\boldsymbol{f}_{2}$ and $\chi_{2}$ have similar expressions as $\boldsymbol{f}_{1}$ and $\chi_{1}$ with $\psi$ remplaced by $\varphi$. It is easy to check that $\left(\boldsymbol{f}_{2}, \chi_{2}\right)$ belongs to $L^{2}\left(\Omega_{R+1}\right) \times H^{1}\left(\Omega_{R+1}\right)$. Since $\Omega^{\prime}$ is of class $\mathscr{C}^{2,1}$, then similar arguments as in [10], allow to prove that the following Stokes problem with mixed boundary conditions

$$
\left\{\begin{array}{l}
-\Delta \boldsymbol{v}+\nabla \eta=\boldsymbol{f}_{2} \text { and } \operatorname{div} \boldsymbol{v}=\chi_{2} \text { in } \Omega_{R+1}, \\
\boldsymbol{v} \cdot \boldsymbol{n}=\boldsymbol{\varphi} \widetilde{\boldsymbol{u}} \cdot \boldsymbol{n} \text { and } 2[\mathbf{D}(\boldsymbol{v}) \boldsymbol{n}]_{\tau}+\alpha \boldsymbol{v}_{\tau}=2[\mathbf{D}(\varphi \widetilde{\boldsymbol{u}}) \boldsymbol{n}]_{\tau}+\alpha \varphi \widetilde{\boldsymbol{u}}_{\tau} \text { on } \Gamma, \\
\boldsymbol{v}=\mathbf{0} \text { on } \partial B_{R+1},
\end{array}\right.
$$

has a unique solution $(\boldsymbol{v}, \eta) \in H^{2}\left(\Omega_{R+1}\right) \times H^{1}\left(\Omega_{R+1}\right)$. The uniqueness of $(\boldsymbol{v}, \eta)$ implies that $(\boldsymbol{v}, \eta)$ is equal to $(\varphi \widetilde{\boldsymbol{u}}, \varphi \widetilde{\pi})$ and therefore $(\varphi \widetilde{\boldsymbol{u}}, \varphi \widetilde{\pi}) \in H^{2}\left(\Omega_{R+1}\right) \times H^{1}\left(\Omega_{R+1}\right)$ which also implies that $(\varphi \widetilde{\boldsymbol{u}}, \varphi \widetilde{\pi})$ belongs to $W_{k+1}^{2}\left(\mathbb{R}^{3}\right) \times W_{k+1}^{1}\left(\mathbb{R}^{3}\right)$.

Consequently, the pair $(\boldsymbol{u}, \pi)$ belongs to $W_{k+1}^{2}(\Omega) \times W_{k+1}^{1}(\Omega)$ and this ends the proof. 
We now look for strong solutions for the case $k>0$. Note that so far, the use of Theorem 2.4 was straightforward because when $k \leq 0$ the compatibility condition (2.10) does not appear since $N_{k-1}$ is reduced to $\{0,0\}$. This is not anymore the case when $k>0$. Thus, to be able to still apply Theorem 2.4, we need to find appropriate extensions of the functions of interest.

Theorem 3.7. Suppose that $\Omega$ is of class $\mathscr{C}^{2,1}$ and let $k>0$ be an integer. Assume that $\boldsymbol{f} \in W_{k+1}^{0}(\Omega), g \in H^{3 / 2}(\Gamma)$, $\chi \in W_{k+1}^{1}(\Omega)$ and $\boldsymbol{h} \in H^{1 / 2}(\Gamma)$ satisfying (3.1). Assume moreover that the following compatibility condition is satisfied

$$
\forall(\boldsymbol{\xi}, \eta) \in \mathscr{N}_{-k}(\Omega), \quad \int_{\Omega} \boldsymbol{f} \cdot \boldsymbol{\xi} d \boldsymbol{x}-\int_{\Omega} \chi \eta d \boldsymbol{x}=\langle g, 2[\boldsymbol{D}(\boldsymbol{\xi}) \boldsymbol{n}] \cdot \boldsymbol{n}-\eta\rangle_{\Gamma}-\langle\boldsymbol{h}, \boldsymbol{\xi}\rangle_{\Gamma}
$$

Then, problem $\left(\mathscr{S}_{T}\right)$ has a unique solution $(\boldsymbol{u}, \pi) \in W_{k+1}^{2}(\Omega) \times W_{k+1}^{1}(\Omega)$ and we have the following estimate:

$$
\|\boldsymbol{u}\|_{W_{k+1}^{2}(\Omega)}+\|\pi\|_{W_{k+1}^{1}(\Omega)} \leq C\left(\|\boldsymbol{f}\|_{W_{k+1}^{0}(\Omega)}+\|\boldsymbol{h}\|_{H^{1 / 2}(\Gamma)}+\|\chi\|_{W_{k+1}^{1}(\Omega)}+\|g\|_{H^{3 / 2}(\Gamma)}\right) .
$$

Proof. Observe first that the uniqueness is a straightforward consequence of Proposition 3.5. We now divide the proof of the theorem into several parts.

- Compatibility condition. In this part, we prove that (3.18) is a necessary condition. Let $(\xi, \eta)$ be in $\mathscr{N}_{-k}(\Omega)$. For any $(\boldsymbol{\varphi}, \psi) \in \mathscr{D}(\bar{\Omega}) \times \mathscr{D}(\bar{\Omega})$, using identity (2.15) and the Green formula (2.7), we have

$$
\int_{\Omega}[(-\Delta \boldsymbol{\varphi}+\nabla \psi) \cdot \boldsymbol{\xi}-\eta \operatorname{div} \boldsymbol{\varphi}] d \boldsymbol{x}=2 \int_{\Omega} \mathbf{D}(\boldsymbol{\varphi}): \nabla \boldsymbol{\xi} d \boldsymbol{x}-2\langle\mathbf{D}(\boldsymbol{\varphi}) \boldsymbol{n}, \boldsymbol{\xi}\rangle_{\Gamma}+\int_{\Omega} \boldsymbol{\varphi} \cdot \nabla \eta d \boldsymbol{x}-\langle\boldsymbol{\varphi} \cdot \boldsymbol{n}, \eta\rangle_{\Gamma}
$$

Observe that we have

$$
\int_{\Omega} \mathbf{D}(\boldsymbol{\varphi}): \nabla \boldsymbol{\xi} d \boldsymbol{x}=\int_{\Omega} \nabla \boldsymbol{\varphi}: \mathbf{D}(\boldsymbol{\xi}) d \boldsymbol{x}
$$

and using again (2.7), we get

$$
2 \int_{\Omega} \mathbf{D}(\boldsymbol{\varphi}): \nabla \boldsymbol{\xi} d \boldsymbol{x}=-\int_{\Omega} \boldsymbol{\varphi} \cdot \Delta \boldsymbol{\xi} d \boldsymbol{x}+2\langle\boldsymbol{D}(\boldsymbol{\xi}) \boldsymbol{n}, \boldsymbol{\varphi}\rangle_{\Gamma} .
$$

Combining this equality with (3.20) and recalling that the pair $(\boldsymbol{\xi}, \eta)$ belongs to $\mathscr{N}_{-k}(\Omega)$, we obtain

$$
\int_{\Omega}[(-\Delta \boldsymbol{\varphi}+\nabla \psi) \cdot \boldsymbol{\xi}-\eta \operatorname{div} \boldsymbol{\varphi}] d \boldsymbol{x}=-2\langle\mathbf{D}(\boldsymbol{\varphi}) \boldsymbol{n}, \boldsymbol{\xi}\rangle_{\Gamma}+2\langle\mathbf{D}(\boldsymbol{\xi}) \boldsymbol{n}, \boldsymbol{\varphi}\rangle_{\Gamma}-\langle\boldsymbol{\varphi} \cdot \boldsymbol{n}, \eta\rangle_{\Gamma}
$$

Now, on the one hand, because $\boldsymbol{\xi} \cdot \boldsymbol{n}=0$ on $\Gamma$, we can write

$$
2\langle\mathbf{D}(\boldsymbol{\varphi}) \boldsymbol{n}, \boldsymbol{\xi}\rangle_{\Gamma}=2\left\langle[\mathbf{D}(\boldsymbol{\varphi}) \boldsymbol{n}]_{\tau}, \boldsymbol{\xi}_{\tau}\right\rangle_{\Gamma}=\left\langle 2[\mathbf{D}(\boldsymbol{\varphi}) \boldsymbol{n}]_{\tau}+\alpha \boldsymbol{\varphi}_{\tau}, \boldsymbol{\xi}_{\tau}\right\rangle_{\Gamma}-\left\langle\alpha \boldsymbol{\varphi}_{\tau}, \boldsymbol{\xi}_{\tau}\right\rangle
$$

On the other hand, because $2[\mathbf{D}(\boldsymbol{\xi}) \boldsymbol{n}]_{\tau}+\alpha \boldsymbol{\xi}_{\tau}=\mathbf{0}$, we can first write

$$
\begin{aligned}
2\langle\mathbf{D}(\boldsymbol{\xi}) \boldsymbol{n}, \boldsymbol{\varphi}\rangle_{\Gamma} & =2\left\langle[\mathbf{D}(\boldsymbol{\xi}) \boldsymbol{n}]_{\tau}, \boldsymbol{\varphi}_{\tau}\right\rangle_{\Gamma}+2\langle([\mathbf{D}(\boldsymbol{\xi}) \boldsymbol{n}] \cdot \boldsymbol{n}) \boldsymbol{n},(\boldsymbol{\varphi} \cdot \boldsymbol{n}) \boldsymbol{n}\rangle_{\Gamma} \\
& =-\left\langle\alpha \boldsymbol{\xi}_{\tau}, \boldsymbol{\varphi}_{\tau}\right\rangle_{\Gamma}+2\langle([\mathbf{D}(\boldsymbol{\xi}) \boldsymbol{n}] \cdot \boldsymbol{n}) \boldsymbol{n},(\boldsymbol{\varphi} \cdot \boldsymbol{n}) \boldsymbol{n}\rangle_{\Gamma} .
\end{aligned}
$$

Next, because $\boldsymbol{n}$ is normalized and belongs to $W^{1, \infty}(\Gamma)$, we have the relation

$$
2\langle([\mathbf{D}(\boldsymbol{\xi}) \boldsymbol{n}] \cdot \boldsymbol{n}) \boldsymbol{n},(\boldsymbol{\varphi} \cdot \boldsymbol{n}) \boldsymbol{n}\rangle_{\Gamma}=2\langle[\mathbf{D}(\boldsymbol{\xi}) \boldsymbol{n}] \cdot \boldsymbol{n}, \boldsymbol{\varphi} \cdot \boldsymbol{n}\rangle_{\Gamma}
$$

As a result, we have

$$
2\langle\mathbf{D}(\boldsymbol{\xi}) \boldsymbol{n}, \boldsymbol{\varphi}\rangle_{\Gamma}=-\left\langle\alpha \boldsymbol{\xi}_{\tau}, \boldsymbol{\varphi}_{\tau}\right\rangle_{\Gamma}+\langle 2[\mathbf{D}(\boldsymbol{\xi}) \boldsymbol{n}] \cdot \boldsymbol{n}, \boldsymbol{\varphi} \cdot \boldsymbol{n}\rangle_{\Gamma} .
$$

Plugging (3.22) and (3.24) in (3.21) leads to

$$
\int_{\Omega}[(-\Delta \boldsymbol{\varphi}+\nabla \psi) \cdot \boldsymbol{\xi}-\eta \operatorname{div} \boldsymbol{\varphi}] d \boldsymbol{x}=\langle\boldsymbol{\varphi} \cdot \boldsymbol{n}, 2[\mathbf{D}(\boldsymbol{\xi}) \boldsymbol{n}] \cdot \boldsymbol{n}-\eta\rangle_{\Gamma}-\left\langle 2[\mathbf{D}(\boldsymbol{\varphi}) \boldsymbol{n}]_{\tau}+\alpha \boldsymbol{\varphi}_{\tau}, \boldsymbol{\xi}_{\tau}\right\rangle_{\Gamma}
$$

Then, for each $(\boldsymbol{\xi}, \eta) \in \mathscr{N}_{-k}(\Omega)$, the Green's formula (3.25) holds for any pair $(\boldsymbol{\varphi}, \psi) \in W_{k+1}^{2}(\Omega) \times W_{k+1}^{1}(\Omega)$ by density. In particular, if $(\boldsymbol{u}, \pi) \in W_{k+1}^{2}(\Omega) \times W_{k+1}^{1}(\Omega)$ is a solution of $\left(\mathscr{S}_{T}\right)$, then (3.18) holds.

- EXISTENCE. Here we prove that problem $\left(\mathscr{S}_{T}\right)$ has a solution $(\boldsymbol{u}, \pi)$ that belongs to $\in W_{k}^{1}(\Omega) \times W_{k}^{0}(\Omega)$. The proof is made of two steps. 
Step 1. The case $g=0$.

Since $k>0$, then $(\boldsymbol{f}, \chi)$ belongs to $W_{1}^{0}(\Omega) \times W_{1}^{1}(\Omega)$. Thanks to Theorem 3.6, problem $\left(\mathscr{S}_{T}\right)$ has a solution $(\boldsymbol{u}, \pi) \in W_{1}^{2}(\Omega) \times W_{1}^{1}(\Omega)$. It remains now to prove that $(\boldsymbol{u}, \pi)$ belongs to $W_{k}^{1}(\Omega) \times W_{k}^{0}(\Omega)$. To that end, we shall use again properties of the Stokes problem in the whole space $\mathbb{R}^{3}$. As we mention before, we first need appropriate extensions of $\boldsymbol{u}$ and $\pi$ defined in $\mathbb{R}^{3}$. So let us consider the following Stokes problem in the bounded domain $\Omega^{\prime}$ :

$$
\left\{\begin{array}{l}
-\Delta \boldsymbol{u}^{\prime}+\nabla \pi^{\prime}=\mathbf{0} \text { and } \operatorname{div} \boldsymbol{u}^{\prime}=0 \text { in } \Omega^{\prime}, \\
\boldsymbol{u}^{\prime}=\boldsymbol{u} \text { on } \Gamma .
\end{array}\right.
$$

Since $\boldsymbol{u} \cdot \boldsymbol{n}=0$, problem (3.26) has a solution $\left(\boldsymbol{u}^{\prime}, \pi^{\prime}\right) \in H^{2}\left(\Omega^{\prime}\right) \times H^{1}\left(\Omega^{\prime}\right)$ (see for instance [6] or [24]). Set now

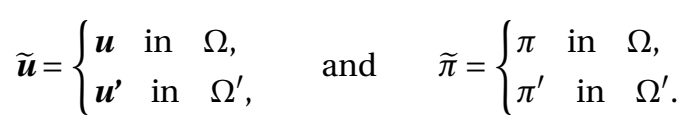

Then clearly, the pair $(\widetilde{\boldsymbol{u}}, \widetilde{\pi})$ belongs to $W_{0}^{1}\left(\mathbb{R}^{3}\right) \times W_{1}^{1}\left(\mathbb{R}^{3}\right)$. The goal is now to identify $(\widetilde{\boldsymbol{u}}, \widetilde{\pi})$ with a solution of the Stokes problem in $\mathbb{R}^{3}$ that belongs to $W_{k}^{1}(\Omega) \times W_{k}^{0}(\Omega)$. Let us set

$$
\boldsymbol{F}=-\Delta \widetilde{\boldsymbol{u}}+\nabla \widetilde{\pi} \quad \text { and } \quad e=\operatorname{div} \widetilde{\boldsymbol{u}} .
$$

In order to apply Theorem 2.4 with data $\boldsymbol{F}$ and $e$, we need to show that $(\boldsymbol{F}, e)$ belongs to $W_{k}^{-1}\left(\mathbb{R}^{3}\right) \times$ $W_{k}^{0}\left(\mathbb{R}^{3}\right)$ and satisfies (2.10) . For any $\boldsymbol{\varphi} \in \mathscr{D}\left(\mathbb{R}^{3}\right)$, we have:

$$
\begin{aligned}
\langle\boldsymbol{F}, \boldsymbol{\varphi}\rangle_{\mathscr{D}^{\prime}\left(\mathbb{R}^{3}\right) \times \mathscr{D}\left(\mathbb{R}^{3}\right)} & =\langle-\Delta \widetilde{\boldsymbol{u}}+\nabla \widetilde{\pi}, \boldsymbol{\varphi}\rangle_{\mathscr{D}^{\prime}\left(\mathbb{R}^{3}\right) \times \mathscr{D}\left(\mathbb{R}^{3}\right)}=-\int_{\mathbb{R}^{3}} \tilde{\boldsymbol{u}} \cdot \Delta \boldsymbol{\varphi} d \boldsymbol{x}-\int_{\mathbb{R}^{3}} \tilde{\pi} \operatorname{div} \boldsymbol{\varphi} d \boldsymbol{x} \\
& =\underbrace{-\int_{\Omega} \boldsymbol{u} \cdot \Delta \boldsymbol{\varphi} d \boldsymbol{x}-\int_{\Omega} \pi \operatorname{div} \boldsymbol{\varphi} d \boldsymbol{x}}_{I_{1}}-\underbrace{\int_{\Omega^{\prime}} \boldsymbol{u} \cdot \Delta \boldsymbol{\varphi} d \boldsymbol{x}-\int_{\Omega^{\prime}} \pi^{\prime} \operatorname{div} \boldsymbol{\varphi} d \boldsymbol{x}}_{I_{2}} .
\end{aligned}
$$

On the one hand, in $\Omega$, using identity (2.15) and the Green formula (2.7), we can write:

$$
\begin{aligned}
I_{1} & =\int_{\Omega} \boldsymbol{u} \cdot(\nabla(\operatorname{div} \boldsymbol{\varphi})-2 \operatorname{div} \mathbf{D}(\boldsymbol{\varphi})) d \boldsymbol{x}-\int_{\Omega} \pi \operatorname{div} \boldsymbol{\varphi} d \boldsymbol{x} \\
& =-\int_{\Omega} \operatorname{div} \boldsymbol{u} \operatorname{div} \boldsymbol{\varphi} d \boldsymbol{x}+\langle\boldsymbol{u} \cdot \boldsymbol{n}, \operatorname{div} \boldsymbol{\varphi}\rangle_{\Gamma}+2 \int_{\Omega} \nabla \boldsymbol{u}: \mathbf{D}(\boldsymbol{\varphi}) d \boldsymbol{x}-2\langle\mathbf{D}(\boldsymbol{\varphi}) \boldsymbol{n}, \boldsymbol{u}\rangle_{\Gamma}-\int_{\Omega} \pi \operatorname{div} \boldsymbol{\varphi} d \boldsymbol{x} .
\end{aligned}
$$

Using the relation

$$
\int_{\Omega} \nabla \boldsymbol{u}: \mathbf{D}(\boldsymbol{\varphi}) d \boldsymbol{x}=\int_{\Omega} \mathbf{D}(\boldsymbol{u}): \nabla \boldsymbol{\varphi} d \boldsymbol{x}
$$

and again (2.15), (2.7) and recalling that $g=0$, we arrive at:

$$
I_{1}=\int_{\Omega} \boldsymbol{f} \cdot \boldsymbol{\varphi} d \boldsymbol{x}+2\langle\mathbf{D}(\boldsymbol{u}) \boldsymbol{n}, \boldsymbol{\varphi}\rangle_{\Gamma}-\langle\boldsymbol{\varphi} \cdot \boldsymbol{n}, \chi\rangle_{\Gamma}-2\langle\mathbf{D}(\boldsymbol{\varphi}) \boldsymbol{n}, \boldsymbol{u}\rangle_{\Gamma}-\langle\boldsymbol{\varphi} \cdot \boldsymbol{n}, \pi\rangle_{\Gamma} .
$$

On the other hand as previously, in $\Omega^{\prime}$, we can write;

$$
\begin{aligned}
I_{2} & =\int_{\Omega^{\prime}} \boldsymbol{u}^{\prime} \cdot(\nabla(\operatorname{div} \boldsymbol{\varphi})-2 \operatorname{div} \mathbf{D}(\boldsymbol{\varphi})) d \boldsymbol{x}-\int_{\Omega^{\prime}} \pi^{\prime} \operatorname{div} \boldsymbol{\varphi} d \boldsymbol{x} \\
& =-\int_{\Omega^{\prime}} \operatorname{div} \boldsymbol{u}^{\prime} \operatorname{div} \boldsymbol{\varphi} d \boldsymbol{x}-\left\langle\boldsymbol{u}^{\prime} \cdot \boldsymbol{n}, \operatorname{div} \boldsymbol{\varphi}\right\rangle_{\Gamma}+2 \int_{\Omega^{\prime}} \nabla \boldsymbol{u}^{\prime}: \mathbf{D}(\varphi) d \boldsymbol{x}+2\left\langle\boldsymbol{u}^{\prime}, \mathbf{D}(\boldsymbol{\varphi}) \boldsymbol{n}\right\rangle_{\Gamma}-\int_{\Omega^{\prime}} \pi \operatorname{div} \boldsymbol{\varphi} d \boldsymbol{x} \\
& =-2\left\langle\mathbf{D}\left(\boldsymbol{u}^{\prime}\right) \boldsymbol{n}, \boldsymbol{\varphi}\right\rangle_{\Gamma}+2\left\langle\mathbf{D}(\boldsymbol{\varphi}) \boldsymbol{n}, \boldsymbol{u}^{\prime}\right\rangle_{\Gamma}+\left\langle\boldsymbol{\varphi} \cdot \boldsymbol{n}, \pi^{\prime}\right\rangle_{\Gamma} .
\end{aligned}
$$

Therefore, denoting by $\widetilde{\boldsymbol{f}} \in W_{k+1}^{0}\left(\mathbb{R}^{3}\right)$ the extension of $\boldsymbol{f}$ by zero in $\Omega^{\prime}$, we deduce that for any $\boldsymbol{\varphi} \in \mathscr{D}\left(\mathbb{R}^{3}\right)$,

$$
\langle\boldsymbol{F}, \boldsymbol{\varphi}\rangle_{\mathscr{D}^{\prime}\left(\mathbb{R}^{3}\right) \times \mathscr{D}\left(\mathbb{R}^{3}\right)}=\int_{\mathbb{R}^{3}} \tilde{\boldsymbol{f}} \cdot \boldsymbol{\varphi} d \boldsymbol{x}+2\left\langle\mathbf{D}(\boldsymbol{u}) \boldsymbol{n}-\mathbf{D}\left(\boldsymbol{u}^{\prime}\right) \boldsymbol{n}, \boldsymbol{\varphi}\right\rangle_{\Gamma}+\left\langle\boldsymbol{\varphi} \cdot \boldsymbol{n}, \pi^{\prime}-\pi\right\rangle_{\Gamma}-\langle\boldsymbol{\varphi} \cdot \boldsymbol{n}, \chi\rangle_{\Gamma} .
$$

Since $\mathscr{D}\left(\mathbb{R}^{3}\right)$ is dense in $W_{-k}^{1}\left(\mathbb{R}^{3}\right)$ and $\tilde{\boldsymbol{f}}$ belongs to $W_{k+1}^{0}\left(\mathbb{R}^{3}\right)$, then (3.28) is still valid for any $\boldsymbol{\varphi} \in$ $W_{-k}^{1}\left(\mathbb{R}^{3}\right)$ which implies that $\boldsymbol{F}$ belongs to $W_{k}^{-1}\left(\mathbb{R}^{3}\right)$. 
Now for any $\phi \in \mathscr{D}\left(\mathbb{R}^{3}\right)$, we have

$$
\begin{aligned}
\langle e, \phi\rangle_{\mathscr{D}^{\prime}\left(\mathbb{R}^{3}\right) \times \mathscr{D}\left(\mathbb{R}^{3}\right)} & =\langle\operatorname{div} \widetilde{\boldsymbol{u}}, \phi\rangle_{\mathscr{D}^{\prime}\left(\mathbb{R}^{3}\right) \times \mathscr{D}\left(\mathbb{R}^{3}\right)}=-\int_{\mathbb{R}^{3}} \widetilde{\boldsymbol{u}} \cdot \nabla \phi d \boldsymbol{x} \\
& =\int_{\Omega} \operatorname{div} \boldsymbol{u} \phi d \boldsymbol{x}+\int_{\Omega^{\prime}} \operatorname{div} \boldsymbol{u}^{\prime} \phi d \boldsymbol{x}-\langle\boldsymbol{u} \cdot \boldsymbol{n}, \phi\rangle_{\Gamma}+\left\langle\boldsymbol{u}^{\prime} \cdot \boldsymbol{n}, \phi\right\rangle_{\Gamma} \\
& =\int_{\Omega} \chi \phi d \boldsymbol{x} .
\end{aligned}
$$

Due to the density of $\mathscr{D}\left(\mathbb{R}^{3}\right)$ in $W_{-k}^{0}\left(\mathbb{R}^{3}\right)$, we obtain

$$
\langle e, \phi\rangle=\int_{\Omega} \chi \phi d x, \quad \forall \phi \in W_{-k}^{0}\left(\mathbb{R}^{3}\right),
$$

which implies that $e$ belongs to $W_{k}^{0}\left(\mathbb{R}^{3}\right)$.

As a result, we have proved that $(\boldsymbol{F}, e)$ belongs to $W_{k}^{-1}\left(\mathbb{R}^{3}\right) \times W_{k}^{0}\left(\mathbb{R}^{3}\right)$. Let us now prove that $\boldsymbol{F}$ and $e$ satisfy (2.10), which in view of (3.28) and (3.29), amounts to prove that for any $(\boldsymbol{\lambda}, \mu)$ in $N_{k-1}$

$$
\int_{\mathbb{R}^{3}} \tilde{\boldsymbol{f}} \cdot \boldsymbol{\lambda} d \boldsymbol{x}-\int_{\Omega} \chi \mu d \boldsymbol{x}+2\left\langle\mathbf{D}(\boldsymbol{u}) \boldsymbol{n}-\mathbf{D}\left(\boldsymbol{u}^{\prime}\right) \boldsymbol{n}, \boldsymbol{\lambda}\right\rangle_{\Gamma}+\left\langle\boldsymbol{\lambda} \cdot \boldsymbol{n}, \pi^{\prime}-\pi\right\rangle_{\Gamma}-\langle\boldsymbol{\lambda} \cdot \boldsymbol{n}, \chi\rangle_{\Gamma}=0 .
$$

So let $(\boldsymbol{\lambda}, \mu) \in N_{k-1}$ and let $(\boldsymbol{v}(\boldsymbol{\lambda}), \theta(\boldsymbol{\lambda}))$ be in $W_{0}^{1}(\Omega) \times L^{2}(\Omega)$ such that the pair $(\boldsymbol{v}(\boldsymbol{\lambda})-\boldsymbol{\lambda}, \theta(\boldsymbol{\lambda})-\mu)$ belongs to $\mathscr{N}_{-k}(\Omega)$. Note that thanks to Theorem 3.6, the pair $(\boldsymbol{\nu}(\boldsymbol{\lambda}), \theta(\boldsymbol{\lambda}))$ also belongs to $W_{1}^{2}(\Omega) \times$ $W_{1}^{1}(\Omega)$.

Now, for any $(\boldsymbol{\nu}, \theta) \in W_{1}^{2}(\Omega) \times W_{1}^{1}(\Omega)$ such that $\operatorname{div} \boldsymbol{v}=0$, computations in $\Omega$ yield

$$
\begin{aligned}
& \int_{\Omega} \boldsymbol{f} \cdot \boldsymbol{v} d \boldsymbol{x}=\int_{\Omega}(-\Delta \boldsymbol{u}+\nabla \pi) \cdot \boldsymbol{v} d \boldsymbol{x} \\
& =2 \int_{\Omega} \nabla \boldsymbol{u}: \mathbf{D}(\boldsymbol{v}) d \boldsymbol{x}+\langle\boldsymbol{v} \cdot \boldsymbol{n}, \chi\rangle_{\Gamma}-2\langle\mathbf{D}(\boldsymbol{u}) \boldsymbol{n}, \boldsymbol{v}\rangle_{\Gamma}+\langle\boldsymbol{v} \cdot \boldsymbol{n}, \pi\rangle_{\Gamma} \\
& =-\int_{\Omega} \Delta \boldsymbol{v} \cdot \boldsymbol{u} d \boldsymbol{x}+\langle\boldsymbol{v} \cdot \boldsymbol{n}, \chi\rangle_{\Gamma}+2\langle\mathbf{D}(\boldsymbol{v}) \boldsymbol{n}, \boldsymbol{u}\rangle_{\Gamma}-2\langle\mathbf{D}(\boldsymbol{u}) \boldsymbol{n}, \boldsymbol{v}\rangle_{\Gamma}+\langle\boldsymbol{v} \cdot \boldsymbol{n}, \pi\rangle_{\Gamma} \\
& =\int_{\Omega}(-\Delta \boldsymbol{v}+\nabla \theta) \cdot \boldsymbol{u} d \boldsymbol{x}+\int_{\Omega} \chi \theta d \boldsymbol{x}+\langle\boldsymbol{v} \cdot \boldsymbol{n}, \chi\rangle_{\Gamma}+2\langle\mathbf{D}(\boldsymbol{v}) \boldsymbol{n}, \boldsymbol{u}\rangle_{\Gamma}-2\langle\mathbf{D}(\boldsymbol{u}) \boldsymbol{n}, \boldsymbol{v}\rangle_{\Gamma}+\langle\boldsymbol{v} \cdot \boldsymbol{n}, \pi\rangle_{\Gamma} .
\end{aligned}
$$

In particular we have

$\int_{\Omega} \boldsymbol{f} \cdot \boldsymbol{v}(\boldsymbol{\lambda}) d \boldsymbol{x}-\int_{\Omega} \chi \theta(\boldsymbol{\lambda}) d \boldsymbol{x}-\langle\boldsymbol{v}(\boldsymbol{\lambda}) \cdot \boldsymbol{n}, \chi\rangle_{\Gamma}-2\langle\mathbf{D}(\boldsymbol{v}(\boldsymbol{\lambda})) \boldsymbol{n}, \boldsymbol{u}\rangle_{\Gamma}+2\langle\mathbf{D}(\boldsymbol{u}) \boldsymbol{n}, \boldsymbol{v}(\boldsymbol{\lambda})\rangle_{\Gamma}-\langle\boldsymbol{v}(\boldsymbol{\lambda}) \cdot \boldsymbol{n}, \pi\rangle_{\Gamma}=0$.

Now making the difference between (3.31) and (3.18) with $\boldsymbol{\xi}=\boldsymbol{\nu}(\boldsymbol{\lambda})-\boldsymbol{\lambda}$ and $\eta=\theta(\boldsymbol{\lambda})-\mu$ and recalling that $\boldsymbol{v}(\boldsymbol{\lambda}) \cdot \boldsymbol{n}=\boldsymbol{\lambda} \cdot \boldsymbol{n}$ on $\Gamma$, yields

$\int_{\Omega} \boldsymbol{f} \boldsymbol{\lambda} d \boldsymbol{x}-\int_{\Omega} \chi \mu d \boldsymbol{x}-\langle\boldsymbol{v}(\boldsymbol{\lambda})-\boldsymbol{\lambda}, \boldsymbol{h}\rangle_{\Gamma}-\langle\boldsymbol{\lambda} \cdot \boldsymbol{n}, \chi\rangle_{\Gamma}-2\langle\mathbf{D}(\boldsymbol{v}(\boldsymbol{\lambda})) \boldsymbol{n}, \boldsymbol{u}\rangle_{\Gamma}+2\langle\mathbf{D}(\boldsymbol{u}) \boldsymbol{n}, \boldsymbol{v}(\boldsymbol{\lambda})\rangle_{\Gamma}-\langle\boldsymbol{\lambda} \cdot \boldsymbol{n}, \pi\rangle_{\Gamma}=0$.

Next, computations on $\Omega^{\prime}$ yield

$$
\begin{aligned}
& \int_{\Omega^{\prime}}\left(-\Delta \boldsymbol{u}^{\prime}+\nabla \pi^{\prime}\right) \cdot \boldsymbol{\lambda} d \boldsymbol{x}=0 \\
& =-\int_{\Omega^{\prime}} \boldsymbol{u}^{\prime} \cdot \Delta \boldsymbol{\lambda} d \boldsymbol{x}+2\left\langle\mathbf{D}\left(\boldsymbol{u}^{\prime}\right) \boldsymbol{n}, \boldsymbol{\lambda}\right\rangle_{\Gamma}-2\left\langle\mathbf{D}(\boldsymbol{\lambda}) \boldsymbol{n}, \boldsymbol{u}^{\prime}\right\rangle_{\Gamma}-\left\langle\boldsymbol{\lambda} \cdot \boldsymbol{n}, \pi^{\prime}\right\rangle_{\Gamma} .
\end{aligned}
$$

The fact that $\boldsymbol{u}=\boldsymbol{u}^{\prime}$ on $\Gamma$, implies

$$
\int_{\Omega^{\prime}} \boldsymbol{u}^{\prime} \cdot \Delta \boldsymbol{\lambda} d \boldsymbol{x}=\int_{\Omega^{\prime}} \boldsymbol{u}^{\prime} \cdot \nabla \mu d \boldsymbol{x}=-\int_{\Omega^{\prime}} \mu \operatorname{div} \boldsymbol{u}^{\prime} d \boldsymbol{x}+\left\langle\boldsymbol{u}^{\prime} \cdot \boldsymbol{n}, \mu\right\rangle_{\Gamma}=0
$$

and we deduce that

$$
2\left\langle\mathbf{D}\left(\boldsymbol{u}^{\prime}\right) \boldsymbol{n}, \boldsymbol{\lambda}\right\rangle_{\Gamma}-2\langle\mathbf{D}(\boldsymbol{\lambda}) \boldsymbol{n}, \boldsymbol{u}\rangle_{\Gamma}-\left\langle\boldsymbol{\lambda} \cdot \boldsymbol{n}, \boldsymbol{\pi}^{\prime}\right\rangle_{\Gamma}=0 .
$$


Combining (3.32) and (3.33) yields

$$
\begin{aligned}
& \int_{\mathbb{R}^{3}} \tilde{\boldsymbol{f}} \cdot \boldsymbol{\lambda} d \boldsymbol{x}-\int_{\Omega} \chi \mu d \boldsymbol{x}-\langle\boldsymbol{\lambda} \cdot \boldsymbol{n}, \chi\rangle_{\Gamma}+\left\langle\boldsymbol{\lambda} \cdot \boldsymbol{n}, \pi^{\prime}-\pi\right\rangle_{\Gamma}-2\left\langle\mathbf{D}\left(\boldsymbol{u}^{\prime}\right) \boldsymbol{n}, \boldsymbol{\lambda}\right\rangle_{\Gamma} \\
& +2\langle\mathbf{D}(\boldsymbol{\lambda}) \boldsymbol{n}-\mathbf{D}(\boldsymbol{v}(\boldsymbol{\lambda})) \boldsymbol{n}, \boldsymbol{u}\rangle_{\Gamma}+2\langle\mathbf{D}(\boldsymbol{u}) \boldsymbol{n}, \boldsymbol{v}(\boldsymbol{\lambda})\rangle_{\Gamma}-\langle\boldsymbol{v}(\boldsymbol{\lambda})-\boldsymbol{\lambda}, \boldsymbol{h}\rangle_{\Gamma}=0 .
\end{aligned}
$$

Let us now compute the terms of the second line of (3.34). Due to the fact that $g=0$ on $\Gamma$ and using the Navier boundary condition in (3.13), we have

$$
\begin{aligned}
& 2\langle\mathbf{D}(\boldsymbol{\lambda}) \boldsymbol{n}-\mathbf{D}(\boldsymbol{v}(\boldsymbol{\lambda})) \boldsymbol{n}, \boldsymbol{u}\rangle_{\Gamma}=2\left\langle[\mathbf{D}(\boldsymbol{\lambda}) \boldsymbol{n}]_{\tau}-[\mathbf{D}(\boldsymbol{v}(\boldsymbol{\lambda})) \boldsymbol{n}]_{\tau}, \boldsymbol{u}_{\tau}\right\rangle_{\Gamma} \\
& =\left\langle\alpha\left(\boldsymbol{v}(\boldsymbol{\lambda})_{\tau}-\boldsymbol{\lambda}_{\tau}\right), \boldsymbol{u}_{\tau}\right\rangle_{\Gamma} .
\end{aligned}
$$

Next, using the fact that $\boldsymbol{v}(\boldsymbol{\lambda}) \cdot \boldsymbol{n}=\boldsymbol{\lambda} \cdot \boldsymbol{n}$ on $\Gamma$,

$$
\begin{aligned}
& 2\langle\mathbf{D}(\boldsymbol{u}) \boldsymbol{n}, \boldsymbol{v}(\boldsymbol{\lambda})\rangle_{\Gamma}=2\left\langle[\mathbf{D}(\boldsymbol{u}) \boldsymbol{n}]_{\tau}, \boldsymbol{v}(\boldsymbol{\lambda})_{\tau}\right\rangle_{\Gamma}+2\langle([\mathbf{D}(\boldsymbol{u}) \boldsymbol{n}] \cdot \boldsymbol{n}) \boldsymbol{n},(\boldsymbol{v}(\boldsymbol{\lambda}) \cdot \boldsymbol{n}) \boldsymbol{n}\rangle_{\Gamma} \\
& =2\left\langle[\mathbf{D}(\boldsymbol{u}) \boldsymbol{n}]_{\tau}, \boldsymbol{v}(\boldsymbol{\lambda})_{\tau}\right\rangle_{\Gamma}+2\langle(\mathbf{D}(\boldsymbol{u}) \boldsymbol{n}) \cdot \boldsymbol{n}, \boldsymbol{\lambda} \cdot \boldsymbol{n}\rangle_{\Gamma}
\end{aligned}
$$

Finally, from (3.1), we clearly have

$$
\langle\boldsymbol{v}(\boldsymbol{\lambda})-\boldsymbol{\lambda}, \boldsymbol{h}\rangle_{\Gamma}=\left\langle\boldsymbol{v}(\boldsymbol{\lambda})_{\tau}-\boldsymbol{\lambda}_{\tau}, \boldsymbol{h}\right\rangle_{\Gamma}
$$

Combining these three expressions, we obtain

$$
\begin{aligned}
& 2\langle\mathbf{D}(\boldsymbol{\lambda}) \boldsymbol{n}-\mathbf{D}(\boldsymbol{v}(\boldsymbol{\lambda})) \boldsymbol{n}, \boldsymbol{u}\rangle_{\Gamma}+2\langle\mathbf{D}(\boldsymbol{u}) \boldsymbol{n}, \boldsymbol{v}(\boldsymbol{\lambda})\rangle_{\Gamma}-\langle\boldsymbol{v}(\boldsymbol{\lambda})-\boldsymbol{\lambda}, \boldsymbol{h}\rangle_{\Gamma} \\
& =\left\langle\alpha\left(\boldsymbol{v}(\boldsymbol{\lambda})_{\tau}\right)-\boldsymbol{\lambda}_{\tau}, \boldsymbol{u}_{\tau}\right\rangle_{\Gamma}+2\left\langle[\mathbf{D}(\boldsymbol{u}) \boldsymbol{n}]_{\tau}, \boldsymbol{v}(\boldsymbol{\lambda})_{\tau}\right\rangle_{\Gamma}+2\langle(\mathbf{D}(\boldsymbol{u}) \boldsymbol{n}) \cdot \boldsymbol{n}, \boldsymbol{\lambda} \cdot \boldsymbol{n}\rangle_{\Gamma}-\left\langle\boldsymbol{v}(\boldsymbol{\lambda})_{\tau}-\boldsymbol{\lambda}_{\tau}, \boldsymbol{h}\right\rangle_{\Gamma} \\
& =\left\langle\alpha\left(\boldsymbol{v}(\boldsymbol{\lambda})_{\tau}-\boldsymbol{\lambda}_{\tau}\right), \boldsymbol{u}_{\tau}\right\rangle_{\Gamma}+2\left\langle[\mathbf{D}(\boldsymbol{u}) \boldsymbol{n}]_{\tau}, \boldsymbol{v}(\boldsymbol{\lambda})_{\tau}-\boldsymbol{\lambda}_{\tau}\right\rangle_{\Gamma}+2\langle\mathbf{D}(\boldsymbol{u}) \boldsymbol{n}, \boldsymbol{\lambda}\rangle_{\Gamma}-\left\langle\boldsymbol{v}(\boldsymbol{\lambda})_{\tau}-\boldsymbol{\lambda}_{\tau}, \boldsymbol{h}\right\rangle_{\Gamma} \\
& =\left\langle\boldsymbol{v}(\boldsymbol{\lambda})_{\tau}-\boldsymbol{\lambda}_{\tau}, 2[\mathbf{D}(\boldsymbol{u}) \boldsymbol{n}]_{\tau}+\alpha \boldsymbol{u}_{\tau}\right\rangle_{\Gamma}+2\langle\mathbf{D}(\boldsymbol{u}) \boldsymbol{n}, \boldsymbol{\lambda}\rangle_{\Gamma}-\left\langle\boldsymbol{v}(\boldsymbol{\lambda})_{\tau}-\boldsymbol{\lambda}_{\tau}, \boldsymbol{h}\right\rangle_{\Gamma} \\
& =\left\langle\boldsymbol{v}(\boldsymbol{\lambda})_{\tau}-\boldsymbol{\lambda}_{\tau}, \boldsymbol{h}\right\rangle_{\Gamma}+2\langle\mathbf{D}(\boldsymbol{u}) \boldsymbol{n}, \boldsymbol{\lambda}\rangle_{\Gamma}-\left\langle\boldsymbol{v}(\boldsymbol{\lambda})_{\tau}-\boldsymbol{\lambda}_{\tau}, \boldsymbol{h}\right\rangle_{\Gamma} \\
& =2\langle\mathbf{D}(\boldsymbol{u}) \boldsymbol{n}, \boldsymbol{\lambda}\rangle_{\Gamma} .
\end{aligned}
$$

Plugging this in (3.34) allows to get (3.30).

Therefore it follows from Theorem 2.4 , that there exists a unique solution $(\widetilde{\boldsymbol{z}}, \widetilde{q}) \in\left(W_{k}^{1}\left(\mathbb{R}^{3}\right) \times W_{k}^{0}\left(\mathbb{R}^{3}\right)\right)$ satisfying the following Stokes problem:

$$
-\Delta \widetilde{z}+\nabla \widetilde{q}=\boldsymbol{F} \text { and } \operatorname{div} \widetilde{z}=e \text { in } \mathbb{R}^{3} .
$$

Using (3.27), we obtain

$$
-\Delta(\widetilde{\boldsymbol{z}}-\widetilde{\boldsymbol{u}})+\nabla(\widetilde{q}-\widetilde{\pi})=\mathbf{0} \text { and } \operatorname{div}(\widetilde{\boldsymbol{z}}-\widetilde{\boldsymbol{u}})=0 \text { in } \mathbb{R}^{3} .
$$

It follows that $(\widetilde{\boldsymbol{z}}-\widetilde{\boldsymbol{u}}, \widetilde{q}-\widetilde{\pi})$ belongs to $\left(W_{k}^{1}\left(\mathbb{R}^{3}\right)+W_{0}^{1}\left(\mathbb{R}^{3}\right)\right) \times\left(W_{k}^{0}\left(\mathbb{R}^{3}\right)+L^{2}\left(\mathbb{R}^{3}\right)\right)$ and since $k>0$ then $(\widetilde{\boldsymbol{z}}-\widetilde{\boldsymbol{u}}, \widetilde{q}-\widetilde{\pi})$ also belongs to $W_{0}^{1}\left(\mathbb{R}^{3}\right) \times L^{2}\left(\mathbb{R}^{3}\right)$. Thanks to Proposition 2.3 , we deduce that $\widetilde{\boldsymbol{z}}-\widetilde{\boldsymbol{u}}=\mathbf{0}$ and $\widetilde{q}-\widetilde{\pi}=0$ which imply that the solution $(\boldsymbol{u}, \pi)$ belongs indeed to $W_{k}^{1}(\Omega) \times W_{k}^{0}(\Omega)$.

Step 2. The case $g \neq 0$.

Let $w$ be in $W_{k+1}^{3}(\Omega)$ be such that $\frac{\partial w}{\partial \boldsymbol{n}}=g$ on $\Gamma$. Consider now the following problem:

$$
\left\{\begin{array}{l}
-\Delta z+\nabla \pi=\boldsymbol{f}+\Delta(\nabla w) \text { and } \operatorname{div} z=\chi-\Delta w \text { in } \Omega, \\
\boldsymbol{z} \cdot \boldsymbol{n}=0 \text { and } 2[\mathbf{D}(\boldsymbol{z}) \boldsymbol{n}]_{\tau}+\alpha \boldsymbol{z}_{\tau}=\boldsymbol{K} \text { on } \Gamma
\end{array}\right.
$$

where

$$
\boldsymbol{K}=\boldsymbol{h}-2[\mathbf{D}(\nabla w) \boldsymbol{n}]_{\tau}-\alpha(\nabla w)_{\tau} .
$$

It is clear that $\boldsymbol{K} \in H^{1 / 2}(\Gamma), \boldsymbol{K} \cdot \boldsymbol{n}=0$ on $\Gamma, \boldsymbol{f}+\Delta(\nabla w)$ belongs to $W_{k+1}^{0}(\Omega)$ and $\chi-\Delta w$ belongs to $W_{k+1}^{1}(\Omega)$. According to step 1, problem (3.35) has a solution in $W_{k}^{1}(\Omega) \times W_{k}^{0}(\Omega)$ if and only if

$$
\forall(\boldsymbol{\xi}, \eta) \in \mathscr{N}_{-k}(\Omega), \quad \int_{\Omega}(\boldsymbol{f}+\Delta(\nabla w)) \cdot \boldsymbol{\xi} d \boldsymbol{x}-\int_{\Omega}(\chi-\Delta w) \eta d \boldsymbol{x}=-\langle\boldsymbol{K}, \boldsymbol{\xi}\rangle_{\Gamma}
$$


But if $(\xi, \eta)$ is in $\mathscr{N}_{-k}(\Omega)$, then since $\nabla w \in W_{k+1}^{2}(\Omega)$, we can write (3.25) for the pair $(-\nabla w, 0)$ and we obtain

$$
\int_{\Omega} \Delta(\nabla w) \cdot \boldsymbol{\xi} d \boldsymbol{x}+\int_{\Omega} \Delta w \eta d \boldsymbol{x}=-\langle g, 2[\mathbf{D}(\boldsymbol{\xi}) \boldsymbol{n}] \cdot \boldsymbol{n}-\eta\rangle_{\Gamma}+\left\langle 2[\mathbf{D}(\nabla w) \boldsymbol{n}]_{\tau}+\alpha(\nabla w)_{\tau}, \boldsymbol{\xi}\right\rangle_{\Gamma} .
$$

Combining (3.18) and (3.37) allows to obtain (3.36). Thus setting $\boldsymbol{u}=\boldsymbol{z}+\nabla w \in W_{k}^{1}(\Omega)$, then the pair $(\boldsymbol{u}, \pi) \in W_{k}^{1}(\Omega) \times W_{k}^{0}(\Omega)$ is a solution of $\left(\mathscr{S}_{T}\right)$.

- Regularity. Finally, to prove that the solution $(\boldsymbol{u}, \pi) \in W_{k}^{1}(\Omega) \times W_{k}^{0}(\Omega)$ of $\left(\mathscr{S}_{T}\right)$ established previously, actually belongs to $W_{k+1}^{2}(\Omega) \times W_{k+1}^{1}(\Omega)$, we can proceed as in the proof of Theorem 3.6 with the use of the partition of unity (3.15).

\section{Conclusions}

In this paper we solved the exterior Stokes problem with the Navier slip boundary conditions with a positive friction term. In order to describe the behavior at infinity of the solutions, we set the problem in weighted spaces. Our study is based on a $L^{2}$-theory. We first established the existence and the uniqueness of the variational solution to the problem. The weighted Korn's inequalities that we proved are the cornerstone of this part. Then we proved existence and uniqueness (possibly up to elements of the kernel) of strong solutions to $\left(\mathscr{S}_{T}\right)$ that may have different behaviors at infinity than the variational solution.

In a forthcoming work, we will study $\left(\mathscr{S}_{T}\right)$ in weighted $L^{p}$ spaces in order to be able to tackle the nonlinear problem of the Navier-Stokes equations in exterior domains with the Navier slip boundary conditions.

\section{References}

[1] Y. Achdou and O. Pironneau. Domain decomposition and wall laws. C. R. Acad. Sci. Paris Sér. I Math., 320(5):541-547, 1995.

[2] Y. Achdou, O. Pironneau, and F. Valentin. Effective boundary conditions for laminar flows over periodic rough boundaries. J. Comput. Phys., 147(1):187-218, 1998.

[3] Y. Achdou, O. Pironneau, and F. Valentin. Shape control versus boundary control. In Équations aux dérivées partielles et applications, pages 1-18. Gauthier-Villars, Éd. Sci. Méd. Elsevier, Paris, 1998.

[4] F. Alliot and C. Amrouche. The Stokes problem in $\mathbb{R}^{n}$ : an approach in weighted Sobolev spaces. Math. Models Methods Appl. Sci., 9(5):723-754, 1999.

[5] F. Alliot and C. Amrouche. Weak solutions for the exterior Stokes problem in weighted Sobolev spaces. Math. Methods Appl. Sci., 23(6):575-600, 2000.

[6] C. Amrouche and V. Girault. Decomposition of vector spaces and application to the Stokes problem in arbitrary dimension. Czechoslovak Math. J., 44(119)(1):109-140, 1994.

[7] C. Amrouche, V. Girault, and J. Giroire. Weighted Sobolev spaces for Laplace's equation in $\mathbb{R}^{n}$. J. Math. Pures Appl. (9), 73(6):579-606, 1994.

[8] C. Amrouche, V. Girault, and J. Giroire. Dirichlet and Neumann exterior problems for the $n$-dimensional Laplace operator: an approach in weighted Sobolev spaces. J. Math. Pures Appl. (9), 76(1):55-81, 1997.

[9] C. Amrouche and M. Meslameni. Stokes problem with several types of boundary conditions in an exterior domain. Electron. J. Differential Equations, No. 196, 28 pp, 2013.

[10] C. Amrouche and A. Rejaiba. $\boldsymbol{L}^{p}$-theory for Stokes and Navier-Stokes equations with Navier boundary condition. J. Differential Equations, 256(4):1515-1547, 2014.

[11] C. Amrouche and A. Rejaiba. Navier-Stokes equations with Navier boundary condition. Math. Methods Appl. Sci., 39(17):5091-5112, 2016.

[12] A. Basson and D. Gérard-Varet. Wall laws for fluid flows at a boundary with random roughness. Comm. Pure Appl. Math., 61 (7):941-987, 2008.

[13] H. Beirão Da Veiga. Regularity for Stokes and generalized Stokes systems under nonhomogeneous sliptype boundary conditions. Adv. Differential Equations, 9(9-10):1079-1114, 2004. 
[14] H. Beirão da Veiga. Vorticity and regularity for flows under the Navier boundary condition. Commun. Pure Appl. Anal., 5(4):907-918, 2006.

[15] S. K. Bhowmik, R. Belbaki, T. Z. Boulmezaoud, and S. Mziou. Solving two dimensional second order elliptic equations in exterior domains using the inverted finite elements method. Comput. Math. Appl., 72(9):2315-2333, 2016.

[16] T. Z. Boulmezaoud. Inverted finite elements: a new method for solving elliptic problems in unbounded domains. M2AN Math. Model. Numer. Anal., 39(1):109-145, 2005.

[17] T. Z. Boulmezaoud, K. Kaliche, and N. Kerdid. Inverted finite elements for div-curl systems in the whole space. Adv. Comput. Math., 43(6):1469-1489, 2017.

[18] T. Z. Boulmezaoud, S. Mziou, and T. Boudjedaa. Numerical approximation of second-order elliptic problems in unbounded domains. J. Sci. Comput., 60(2):295-312, 2014.

[19] J. Casado-Díaz, E. Fernández-Cara, and J. Simon. Why viscous fluids adhere to rugose walls: a mathematical explanation. J. Differential Equations, 189(2):526-537, 2003.

[20] P. G. Ciarlet. Mathematical elasticity. Vol. I, volume 20 of Studies in Mathematics and its Applications. North-Holland Publishing Co., Amsterdam, 1988. Three-dimensional elasticity.

[21] P. G. Ciarlet and P. Ciarlet Jr. Another approach to linearized elasticity and a new proof of Korn's inequality. Math. Models Methods Appl. Sci., 15(2):259-271, 2005.

[22] E. Friedmann. The optimal shape of riblets in the viscous sublayer. J. Math. Fluid Mech., 12(2):243-265, 2010.

[23] E. Friedmann and T. Richter. Optimal microstructures drag reducing mechanism of riblets. J. Math. Fluid Mech., 13(3):429-447, 2011.

[24] G. P. Galdi. An introduction to the mathematical theory of the Navier-Stokes equations. Vol. I, volume 38 of Springer Tracts in Natural Philosophy. Springer-Verlag, New York, 1994.

[25] G. P. Galdi and C. G. Simader. Existence, uniqueness and $L^{q}$-estimates for the Stokes problem in an exterior domain. Arch. Rational Mech. Anal., 112(4):291-318, 1990.

[26] G. P. Galdi and C. G. Simader. New estimates for the steady-state Stokes problem in exterior domains with applications to the Navier-Stokes problem. Differential Integral Equations, 7(3-4):847-861, 1994.

[27] D. Gérard-Varet and N. Masmoudi. Relevance of the slip condition for fluid flows near an irregular boundary. Comm. Math. Phys., 295(1):99-137, 2010.

[28] V. Girault. The gradient, divergence, curl and Stokes operators in weighted Sobolev spaces of $\mathbb{R}^{3}$. J. Fac. Sci. Univ. Tokyo Sect. IA Math., 39(2):279-307, 1992.

[29] V. Girault. The Stokes problem and vector potential operator in three-dimensional exterior domains: an approach in weighted Sobolev spaces. Differential Integral Equations, 7(2):535-570, 1994.

[30] V. Girault, J. Giroire, and A. Sequeira. A stream-function-vorticity variational formulation for the exterior Stokes problem in weighted Sobolev spaces. Math. Methods Appl. Sci., 15(5):345-363, 1992.

[31] V. Girault and P. A. Raviart. Finite Element Methods for Navier-Stokes Equations, Theory and Algorithms. Springer-Verlag, 1986.

[32] V. Girault and A. Sequeira. A well-posed problem for the exterior Stokes equations in two and three dimensions. Arch. Rational Mech. Anal., 114(4):313-333, 1991.

[33] J. Giroire. Étude de quelques problèmes aux limites extérieurs et résolution par équations intégrales. Thèse de Doctorat d'État. Université Pierre et Marie Curie, Paris, 1987.

[34] B. Hanouzet. Espaces de Sobolev avec poids. Application au problème de Dirichlet dans un demi espace. Rend. Sem. Mat. Univ. Padova, 46:227-272, 1971.

[35] W. Jäger and A. Mikelić. On the roughness-induced effective boundary conditions for an incompressible viscous flow. J. Differential Equations, 170(1):96-122, 2001.

[36] Daniel D. Joseph and Gordon S. Beavers. Boundary conditions at a naturally permeable wall. J. Fluid Mech., 30:197-207, 1967.

[37] V. A. Kondratiev and O. A. Oleĭnik. Boundary value problems for a system in elasticity theory in unbounded domains. Korn inequalities. Uspekhi Mat. Nauk, 43(5(263)):55-98, 239, 1988. 
[38] H. Kozono and H. Sohr. New a priori estimates for the Stokes equations in exterior domains. Indiana Univ. Math. J., 40(1):1-27, 1991.

[39] H. Kozono and H. Sohr. On a new class of generalized solutions for the Stokes equations in exterior domains. Ann. Scuola Norm. Sup. Pisa Cl. Sci. (4), 19(2):155-181, 1992.

[40] A. Kufner. Weighted Sobolev spaces. A Wiley-Interscience Publication. John Wiley \& Sons Inc., New York, 1985.

[41] J.-L. Lions and E. Magenes. Problèmes aux limites non homogènes et applications. Vol. 1. Travaux et Recherches Mathématiques, No. 17. Dunod, Paris, 1968.

[42] H. Louati, M. Meslameni, and U. Razafison. Weighted $L^{p}$-theory for vector potential operators in threedimensional exterior domains. Math. Methods Appl. Sci., 39(8):1990-2010, 2016.

[43] G. Mulone and F. Salemi. On the existence of hydrodynamic motion in a domain with free boundary type conditions. Meccanica, 18:136-144, 1983.

[44] G. Mulone and F. Salemi. On the hydrodynamic motion in a domain with mixed boundary conditions: existence, uniqueness, stability and linearization principle. Ann. Mat. Pura Appl. (4), 139:147-174, 1985.

[45] C.L.M.H. Navier. Mémoire sur les Lois du Mouvement des fluides. Mem. Acad. Sci. Inst. de France (2), 6:389-440, 1827.

[46] A. Russo and A. Tartaglione. On the Navier problem for the stationary Navier-Stokes equations. J. Differential Equations, 251(9):2387-2408, 2011.

[47] J. Serrin. Mathematical principles of classical fluid mechanics. In Handbuch der Physik (herausgegeben von S. Flügge), Bd. 8/1, Strömungsmechanik I (Mitherausgeber C. Truesdell), pages 125-263. SpringerVerlag, Berlin-Göttingen-Heidelberg, 1959.

[48] V. A. Solonnikov and V. E. Scadilov. A certain boundary value problem for the stationary system of NavierStokes equations. Trudy Mat. Int. Steklov., 125:1515-1547,235, 1973.

[49] M. Specovius-Neugebauer. Exterior Stokes problems and decay at infinity. Math. Methods Appl. Sci., 8(3):351-367, 1986.

[50] M. Specovius-Neugebauer. Weak solutions of the Stokes problem in weighted Sobolev spaces. Acta Appl. Math., 37(1-2):195-203, 1994. 